\title{
XI. Längen von Formeln
}

von Ernst Specker und Georges Wick

Dieser Vortrag besteht aus zwei - unabhängig vaneinander lesbaren Teilen. Grundlage des ersten Teiles ist eine Arbeit von Netschiporuk (Nečiporuk 1966). Grundlage des zweiten Teiles ist ein Artikel von Hodes und Specker (Hodes \& Specker 1968).

Im ersten Teil wird der Grundgedanke der Arbeit von Netschiporuk im Anschluss an einen Artikel von Harper und Savage (Harper \& Savage 1972) als allgemeiner Satz formuliert, und denn auf zwei spezielle Probleme angewandt. Der Kern des allgemeinen Satzes ist das folgende Lemma: Es sei $f$ eine Boole'sche Abbildung in $(k+n)$ Variablen, d.h. f ordne jedem Boole'schen $(k+n)-T u p e l$

$$
\left\langle p_{1}, \ldots, p_{k}, x_{1}, \ldots, x_{n}\right\rangle=\langle\vec{p}, \vec{x}\rangle
$$

einen der Werte 0,1 zu. Für ein solches $f$ sei $e(f, X)$ die Anzahl jener Abbildungen $g$ von $\{0,1\}^{n}$ in $\{0,1\}$, zu denen es ein Boole'sches k-Tupel $\vec{p}$ gibt, sodass für alle Boole'schen n-Tupel $\vec{x}$ gilt: $g(x)=f(\langle\vec{p}, \vec{x}\rangle)$. Ist nun $\varphi$ eine Boole'sche Formel über $0,1,+, \cdot$, und in den Variablen $p_{1}, \ldots, p_{k}, x_{1}, \ldots, x_{n}$, welche $f$ darstellt, und ist $\ell(\varphi, x)$ die Anzahl der mit Vielfachheit gezählten - Variablen $x_{1}, \ldots, x_{n}$ in $\varphi$, so gilt

$$
\frac{\log e(f, X)}{\log 5}<\ell(\varphi, X) \text {. }
$$

Der Buchstabe $X$ in "e $(f, X)$ " und "$\ell(\varphi, X)$ " ist für dieses Lemma ohne Bedeutung, deutet aber die Möglichkeit an, die Menge der Variablen von $f$ beliebig zu zerlegen in "p-Variable" und "x-Variable".

Diese Möglichkeit leitet über vom Lemma zum allgemeinen Satz. Ist nämlich $f$ eine Boole'sche Abbildung in der Variablenmenge $V$, und $V_{1}, \ldots, V_{m}$ eine Partition von $V$, so ergibt die Sumation:

$$
\frac{1}{\log 5} \sum_{i=1}^{m} \log e\left(f, v_{i}\right)<\sum_{i=1}^{m} \ell\left(\varphi, V_{i}\right)=\ell(\varphi, V) \text {. }
$$

Diese Ungleichung ergibt eine untere Schranke für die Länge einer Darstellung der Boole'schen Abbildung $f$.

Eine erste Anwendung des Satzes zeigt, dass die darin angegebene Schranke in einem gewissen Sinne scharf ist. (Vgl. Korollar 1 auf Seite 7). In einem zweiten Korollar (vgl. Seite 10) wird der Satz auf des Heiratsproblem angewandt, ein Beispiel, welches schon im Artikel von 
Harper und Savage in anderer Form behandelt worden ist. Bei diesem Beispiel liegen die untere und die obere Schranke weit auseinander. Zur Vorbereitung auf den zweiten Teil des Vortrags wird gezeigt, dass für eine symmetrische Abbildung $f$ die Summe

$$
\frac{1}{\log 5} \sum_{i} \log e\left(f, V_{i}\right)
$$

höchstens gleich der Anzahl der Variablen aus $V$ ist.

Zum Schluss des ersten Teiles wird der vorgeführte Beweis des Satzes verallgemeinert, und zwar vom Fall der speziellen Basis $0,1,+$, auf den - auch von Netschiporuk betrachteten - Fall einer allgemeinen $\mathrm{Ba}-$ sis. Für Basen, welche Abbildungen mit höchstens q Stellen enthalten, lautet die Ungleichung des Lemmas:

$$
\frac{\log e(f, x)}{\log \left(2^{q}+1\right)}<\ell(\varphi, x) .
$$

Die Verallgemeinerung des Satzes auf Abbildungen über einer beliebigen endlichen Merige (z.B. mit k Elementen) ist im Vortrag nicht durchgeführt. Es lässt sich aber leicht und schnell verifizieren, dass in diesem Fall die Ungleichung des entscheidenden Lemmas wie folgt lauten muss:

$$
\frac{\log e(f, x)}{\log \left(k^{q-2}\left(k^{k}-k\right)^{2}+1\right)}<\ell(\varphi, x) .
$$

Der zweite Teil gibt im Wesentlichen den erwähnten Artikel von Hodes und Specker wieder. Die Beweise wurden etwas modifiziert. Einige Teilschritte wurden vom speziellen Kärper mit dem Universum $\{0,1\}$ auf einen beliebigen endlichen und kommutativen Ring mit Einselement verallgemeinert. Damit soll gezeigt werden, an welchen Teilschritten es liegt, dass die Sätze selbst nicht verallgemeinert werden können.

In etwas nachlässiger Sprechweise kann das Resultat des Artikels von Hodes und Specker so formuliert werden:

Es sei $\varphi$ eine Boole'sche Formel über $0,1,+, \cdot$ und der Variablenmenge $V$. Für eine Teilmenge $U$ von $V$ bezeichne $\varphi / U$ jene Formel, welche aus $\varphi$ durch Ersetzen der Variablen aus $V U$ durch 0 entsteht. Ist dann das VerhäItnis zwischen der Länge von $\varphi$ und $|V|$ genügend klein, so gibt es eine noch relativ grosse Teilmenge $U$ von $V$, sodass $\varphi / U$ äquivalent ist einer Formel der Form

$$
a+b \cdot \prod_{x \in U}(1+x)+c \cdot \sum_{x \in U} x .
$$


Zum Schluss wird dieses Resultat angewandt auf gewisse symmetrische Abbildungen. Da die Formulierung dieser Sätze nicht ganz einfach ist, erwähnen wir hier nur ein unmittelbares korollar:

Es sei $S$ eine Abbildung von $\mathbb{N}$ in $\mathbb{N}$ mit der Eigenschaft, dass jede symmetrische Abbildung $f:\{0,1\}^{n} \Longrightarrow\{0,1\}$ dargestellt werden kann durch eine Formel, deren Länge kleiner ist als $S(n)$. Dann gilt:

$$
\lim \frac{S(n)}{n}=\infty \text {. }
$$

Zum gleichen Thema ist 1975 eine Arbeit erschienen (Fischer. Meyer \& Paterson 1975). Danach lässt sich das obige Korollar folgendermassen verschärfen:

$$
\lim _{n} \frac{S(n) \cdot \log (\log n)}{n \cdot \log n}=\infty
$$

\section{Teil}

Es sei $V$ eine Menge von Variablen; $\Phi(V)$ sei die kleinste Menge $F$ von Formeln, welche die folgenden Eigenschaften hat: Die Formeln 0,1 und die Variablen aus $V$ gehören zu F; mit $\varphi$ und $\psi$ gehären auch $(\varphi+\psi)$ und $(\varphi * \psi) z u F$.

Für eine Formel $\varphi$ aus $\Phi(V)$ und eine Teilmenge $X$ von $V$ sei $\ell(\varphi, X)$ die mit Vielfachheit gezählte - Anzahl der Variablen aus $X$ in $\varphi$, d.h. es sei

$$
\begin{aligned}
& \ell(\varphi, X)=0, \text { falls } \varphi \text { eine der Formeln } 0 \text { oder } 1 \text {, oder } \\
& \text { falls } \varphi \text { eine Variable nicht aus } x \text { ist; } \\
& \begin{aligned}
& \ell(\varphi, X)=1, \text { falls } \varphi \text { eine Variable aus } X \text { ist; } \\
& \ell\left(\left(\varphi_{1} * \varphi_{2}\right), X\right)= \ell\left(\varphi_{1}, X\right)+\ell\left(\varphi_{2}, X\right) \text {, wobei }\left(\varphi_{1}^{*} \varphi_{2}\right) \\
& \text { für }\left(\varphi_{1}+\varphi_{2}\right) \text { oder }\left(\varphi_{1} \cdot \varphi_{2}\right) \text { steht. }
\end{aligned} \\
& \ell(\varphi, V) \text { ist die Länge von } \varphi .
\end{aligned}
$$

Einer formel $\varphi$ aus $\Phi(V)$ ist in bekannter Weise eine Abbildung $\hat{\varphi}$ von $\{0,1\}^{V}$ in $\{0,1\}$ zugeordnet:

Ist $b$ aus $\{0,1\}^{V}, d . h$. b: $V \Longrightarrow\{0,1\}$, so wird $\varphi(b)$ erhalten, indem die Variablen $x$ in $\varphi$ durch $b(x)$ ersetzt werden, und die entstehende Formel ausgewertet wird, wobei + und - als Boole'sche Summe und Produkt in $\{0,1\}$ interpretiert werden. 
Es sei $X$ eine Teilmenge von $V$. Je zwei Abbildungen $b_{1}$, $b_{2}$ mit

$$
\begin{aligned}
& b_{1}: x \Longrightarrow\{0,1\} \\
& b_{2}: v x \Longrightarrow\{0,1\}
\end{aligned}
$$

ist eine Abbildung $\left[b_{1}, b_{2}\right]$ von $V$ in 0,1 zugeordnet, welche definiert ist durch

$$
\begin{aligned}
& {\left[b_{1}, b_{2}\right](x)=b_{1}(x), \text { für } x \text { aus } x,} \\
& {\left[b_{1}, b_{2}\right](x)=b_{2}(x) \text {, für } x \text { aus } V x .}
\end{aligned}
$$

Es sei $f$ eine Abbildung von $\{0,1\}^{V}$ in $\{0,1\}$, und $X$ eine Teilmenge von $V$. Dann sei $E(f, X)$ die folgende Menge von Abbildungen von $(0,1\}$ in $\{0,1\}$ :

Die Abbildung von $\{0,1\}^{X}$ in $\{0,1\}$ gehört genau dann $z u E(f, X)$, wenn es eine Abbildung $c$ von $V X$ in $\{0,1\}$ gibt, sodass für alle Abbildungen $b$ von $x$ in $\{0,1\}$ gilt: $g(b)=f([b, c])$.

$E(f, x)$ besteht somit aus denjenigen Abbildungen von $\{0,1\}^{X}$ in $\{0,1\}$, welche aus der Abbildung $f$ von $\{0,1\}^{V}$ in $\{0,1\}$ erhalten werden, indem die Elemente aus $V \backslash X$ durch werte 0,1 belegt werden.

\section{Lemma}

Es sei $\varphi$ eine Formel aus $\Phi(V), X$ eine Teilmenge von $V$. Dann ist die Anzahl der Elemente von $E(\hat{\varphi}, X)$ höchstens gleich $\frac{1}{2} \cdot\left(5^{\ell(0, X)}+3\right)$.

Zum Beweis ordnen wir zunächst einer Menge $E$ von Abbildungen von $\{0,1\}^{X}$ in $\{0,1\}$ eine Menge $\bar{E}$ von ebensolchen Abbildungen zu gemäss der Vorsohrift:

Díe Abbildung $f$ gehört genau dann zu $\vec{E}$, wenn $f$ eine der beiden konstanten Abbildungen ist, oder wenn es eine Abbildung $g$ in E gibt, sodass die Boole'sche Summe $f+g$ konstant ist ( $d . h$. dass es eine konstante $A b-$ bildung $c$ gibt mit $f=g+c$ ).

Offenbar ist $E \subset \vec{E}$. Für den Beweis des Lemmas zeigen wir mit Induktion nach dem Formelaufbau: Die Anzahl der Elemente von $\bar{E}(\hat{\varphi}, X)$ ist höchstens gleich $\frac{1}{2} \cdot\left(5^{l(0, x)}+3\right)$.

Ist $\varphi$ eine der Formeln 0,1 oder eine Variable, welche nicht zu $X$ gehört, so ist $\ell(\varphi, X)=0$, und $\bar{E}(\hat{\varphi}, X)$ besteht aus den beiden konstanten Abbildungen; es ist aber $\frac{1}{2} \cdot\left(5^{\circ}+3\right)=2$.

Ist $\varphi$ eine Variable aus $X$, so ist $\ell(\varphi, X)=1$, und $\bar{E}(\hat{\varphi}, X)$ besteht aus den 4 Abbildungen, welche durch die Formeln $0,1, x$ und $(1+x)$ dargestelit 
werden; es ist aber $\frac{1}{2} \cdot\left(5^{1}+3\right)=4$.

Es sei nun $\varphi$ eine der beiden Formeln $\left(\varphi_{1} * \varphi_{2}\right),\left(\varphi_{1} \cdot \varphi\right)$; wir fassen die beiden Fälle durch $\left(\varphi_{1} * \varphi_{2}\right)$ zusammen. Es ist $\ell\left(\left(\varphi_{1} * \varphi_{2}\right), X\right)=\ell\left(\varphi_{1}, X\right)+$ $\ell\left(\varphi_{2}, X\right)$. Ist $f$ ein Element van $E(\hat{\varphi}, X)$, so gibt es Elemente $f_{i}$ von $E\left(\tilde{\varphi}_{1}, X\right)$,sodass $f=f_{1} * f_{2}$. (Dabei deutet der Stern hier die Boole'sche Summe oder das Produkt an je nach dem Zeichen in $\left(\varphi_{1}^{*} \varphi_{2}\right)$.) Ist $f$ in $\bar{E}(\hat{\varphi}, X)$, so ist $f$ konstant, oder es gibt Elemente $f_{i}$ in $E\left(\hat{\varphi}_{i}, X\right)$ - und damit auch in $\bar{E}\left(\hat{\varphi}_{i}, x\right)$ - und eine Konstante $c$, sodass $f=\left(f_{1} * f_{2}\right)+c$. Die Anzahl der Elemente von $\bar{E}\left(\hat{\varphi}_{i}, X\right)$ sei gleich $a_{i}$. Zur Abschätzung der Anzahl der Elemente von $\bar{E}(\hat{\varphi}, X)$ unterscheiden wir 4 Fälle (wobel stets $f_{i}$ in $\left.\bar{E}\left(\hat{\varphi}_{i}, X\right)\right)$ :

(1) $f$ ist konstant, oder $f=\left(f_{1} * f_{2}\right)+c$, wobei $f_{1}, f_{2}$ beide kanstant sind.

In diesem Fall ist $f$ konstant; es gibt zwei konstante Abbildungen.

(2) Es ist $f=\left(f_{1} * f_{2}\right)+c$, wobei $f_{1}$ nicht konstant, und $f_{2}$ konstant. Ist * die Summe, so ist $f=f_{1}{ }^{+} c^{\prime}$ ( $c^{\prime}$ kanstant), $d . h$. f ist ein nicht kanstantes Element von $\bar{E}\left(\hat{\varphi}_{1}, x\right)$. Es gibt $a_{1}-2$ solche Elemente. Ist * das Produkt, so ist $f$ konstant, falls $f_{2}$ konstant 0 , oder $f=f_{1}+c$, falls $f_{2}$ konstant $1.0 . h$. $f$ ist ein Element von $\bar{E}\left(\hat{\varphi}_{1}, X\right)$.

Es gibt auch in diesem Fall höchstens $a_{1}-2$ nicht konstante Elemente.

(3) Es ist $f=\left(f_{1} * f_{2}\right)+c$, wobei $f_{1}$ konstant, und $f_{2}$ nicht kanstant. Nicht kanstante Elemente dieser Art gibt es höchstens $a_{2}-2$ (entsprechend dem Fall 2).

(4) Es ist $f=\left(f_{1} * f_{2}\right)+c$, wobei die $f_{i}$ nicht konstante Elemente von $\bar{E}\left(\hat{\varphi}_{i}, x\right)$ sind. Für $f_{i}$ gibt es höchstens $a_{i}-2$ Mäglichkeiten, für o hächstens 2. Die Anzahl der dargesteliten Abbildungen ist höchstens $2 \cdot\left(a_{1}-2\right) \cdot\left(a_{2}-2\right) \cdot$

Zusammenfassend erhalten wir die Abschätzung

$$
\begin{aligned}
\vec{E}(\hat{\varphi}, x) & \leq 2 *\left(a_{1}-2\right)+\left(a_{2}-2\right)+2\left(a_{1}-2\right)\left(a_{2}-2\right) \\
& =\frac{1}{2} \cdot\left(\left(2 a_{1}-3\right)\left(2 a_{2}-3\right)+3\right) .
\end{aligned}
$$

Nach Induktionsvoraussetzung ist

$$
\begin{aligned}
a_{i} & \leq \frac{1}{2} \cdot\left(5^{l\left(\varphi_{i}, x\right)}+3\right), d \cdot h . \\
\left(2 a_{i}-3\right) & \leq 5^{l\left(\varphi_{i}, x\right)} .
\end{aligned}
$$


Daher ist

$$
\begin{aligned}
|\vec{E}(\hat{\varphi}, X)| & \leq \frac{1}{2} \cdot\left(5^{\ell\left(\varphi_{1}, X\right)} \cdot 5^{\ell\left(\varphi_{2}, X\right)}+3\right) \\
& =\frac{1}{2} \cdot\left(5^{\ell\left(\varphi_{1}, X\right)+l\left(\varphi_{2}, X\right)}+3\right) \\
& =\frac{1}{2} \cdot\left(5^{\ell(\varphi, X)}+3\right) .
\end{aligned}
$$

Bemerkung Die angegebene Schrenke ist genau; im Fall $\ell(\varphi, x)=2$ ist sie 14. Durch

$$
\left(c_{0}+c_{1} x_{1}\right)\left(c_{2} * c_{3} x_{2}\right)+c_{4}
$$

werden wirklich 14 Abbildungen dargestellt. Von den total mägliohen 16 Abbildungen fehlen dabei die durch $\left(x_{1}+x_{2}\right)$ und $\left(1+\left(x_{1}+x_{2}\right)\right)$ dargestellten. Wir werden das Lemma in der folgenden form verwenden:

\section{Korollar}

Es sei $\varphi$ eine Formel aus $\Phi(V)$, und $X$ eine Teilmenge von $V$. Ist dann die Anzahl $n$ der Elemente von $E(\hat{\varphi}, X)$ grösser als 2 (d.h. enthält $E(\hat{\varphi}, X)$ eine nicht kanstante Abbildung), so gilt für die Länge von $\varphi$ in Variablen aus $X$ die folgende Abschätzung:

$$
\frac{\log n}{\log 5}<\ell(\varphi, x) \text {. }
$$

Beweis Nach dem Lemma ist $n \leq \frac{1}{2} \cdot\left(5^{\ell(\varphi, X)}+3\right)$. Da $|E(\hat{\varphi}, X)|$ grösser als 2 , ist $0<\ell(\varphi, X)$. Für positive ganze Zahlen $m$ gilt $\frac{1}{2} \cdot\left(5^{m}+3\right)<5^{m}$, und somit ist $\log n<\ell(\varphi, x) \cdot \log 5$.

(Dabei und im folgenden ist mit log stets der Logarithmus zur Basis 2 gemeint.)

\section{Satz von Netschiporuk}

Es sei $V$ eine Menge von Variablen, feine Abbildung von $\{0,1\}^{V}$ in

$\{0,1\}$, $\varphi$ eine Formel aus $\Phi(V)$, welche $f$ darstelit, und $\left\{x_{1}, \ldots x_{m}\right\}$ eine Menge von disjunkten Teilmengen von $V$. Die Anzahl $e_{i}$ der Elemente von $E\left(f, X_{i}\right)$ sei grösser als $2(i=1, \ldots, m)$. Dann gilt

$$
\frac{1}{\log 5} \sum_{i=1} \log 8 e_{i}<\ell(\varphi, V) \text {. }
$$

Beweis Nach dem vorigen Korollar ist

$$
\frac{1}{\log 5} \cdot \log e_{i}<\ell\left(\varphi, x_{i}\right) \quad(i=1, \ldots, m) .
$$

Da die Mengen $x_{1}, \ldots, x_{m}$ disjunkt sind, gilt 


$$
\sum_{i=1}^{m} \ell\left(\varphi, x_{i}\right) \leq \ell(\varphi, v) \text {, }
$$

woraus die Behauptung folgt.

\section{Erste Anwendung}

Es seien $m, n$ naturliche Zahlen grösser als 1 , und $V$ sei die Menge der Variablen $x_{i k}$ mit $1 \leq i \leq m, 1 \leq k \leq n$. Wir definieren eine Abbildung $f$ von $\{0,1\}^{V}$ in $\{0,1\}$ :

Ist b eine Abbildung von $V$ in $\{0,1\}$, so ist genau dann $f(b)=1$, wenn es Zahlen $i, j$ mit $1 \leq i<j \leq m$ gibt, sodass für alle $k$ mit $1 \leq k \leq n$ gilt:

$$
b\left(x_{i k}\right)=b\left(x_{j k}\right) \text {. }
$$

Ist $\varphi$ eine Formel aus $\Phi(V)$, welche f darstellt, so besagt $n$ in enschaulicher Ausdrucksweise, dass in der Matrix

$$
\left(\begin{array}{ccc}
x_{11} & \cdots & x_{1 n} \\
\cdot & & \vdots \\
x_{m 1} & \cdots & x_{m n}
\end{array}\right)
$$

zwei in verschiedenen Zeilen stehende n-Tupel gleich sind.

Korollax 1 Für die Länge einer Formel $\varphi$, welche $f$ möglichst kurz darstelit, gilt

$$
\frac{1}{\log 5} \cdot m \cdot \log \left(2^{n}-1\right)<\ell(\varphi, v)<m^{2} \cdot n \text {. }
$$

Für $m=2^{n / 2}+1$ (bei geradem $n$ ) gilt dann

$$
\frac{1}{36} \cdot \frac{(m \cdot n)^{2}}{\log (m \cdot n)}<\ell(\varphi, v)<\frac{3}{2} \cdot \frac{(m \cdot n)^{2}}{\log (m \cdot n)},
$$

d.h. $\ell(\varphi, v)$ ist bis auf einen Faktor 54 bestimmt.

Zur oberen Schranke: Es sei $\alpha_{i j}$ die folgende Formel:

$$
\prod_{k=1}^{n}\left(x_{i k}+x_{j k}+1\right) \text {; }
$$

d.h. as sei zum beispiel für $n=3 \alpha_{12}$ die Formel

$$
\left(\left(\left(x_{11}+x_{21}\right)+1\right) \cdot\left(\left(\left(x_{12}+x_{22}\right)+1\right) \cdot\left(\left(x_{13}+x_{23}\right)+1\right)\right)\right) .
$$

Offenbar ist genau dann $\vec{\alpha}_{i j}(b)=1$, wenn

$$
b\left(x_{i j}\right)=b\left(x_{j k}\right) \text { für allek. }
$$

$\alpha$ sei die Formel

$$
\left(\prod_{i<j}\left(\alpha_{i j}+1\right)\right)+1
$$


Offenbar ist genau dann $\hat{\alpha}(t)=1$, wenn es Zahlen $i, j$ mit $1 \leq i<j \leq m$ und $\tilde{\alpha}_{i j}(b)=1$ gibt. D.h. a stellt $f$ dar. Für die Länge von $\alpha$ gilt die folgende Abschätzung:

$$
\ell(\alpha, v)=\left(\frac{m}{2}\right) \cdot 2 n=m(m-1) n<m^{2} \cdot n .
$$

Zur unteren Schranke: Es sei für $1 \leq i \leq m$

$$
x_{i}=\left\{x_{i 1}, \ldots, x_{i n}\right\} \text {, }
$$

$\left\{x_{1}, \ldots, x_{m}\right\}$ ist dann eine Menge von disjunkten Teilmengen von $V$. Ist $\varphi$ eine Formel aus $\Phi(V)$, welche $f$ darstellt, und $e_{i}$ die Anzahl der Elemente von $E\left(f, X_{i}\right) \quad(1 \leq i \leq m)$, so ist $2<\theta_{i}$, und deshalb nach dem Satz van Netschiporuk

$$
\frac{1}{\log 5} \cdot \sum_{i=1} \log \varepsilon_{i}<\ell(\varphi, V) .
$$

Zur Abschätzung der Anzahl $e_{1}$ der Elemente von $E\left(f, x_{1}\right)$ - d.h. von $E\left(\hat{\varphi}, X_{1}\right)$ - bedienen wir uns der anschaulichen Ausdrucksweise und unterscheiden 2 Fälle:

(1) Zwei der in den letzten $(m-1)$ Zeilen stehenden n-Tupel sind gleich. Dann sind zwei n-Tupel. in der ganzen Matrix gleich, und die resultierende Abbildung von $\{0,1\}^{X_{1}}$ in $\{0,1\}$ ist die konstante Abbildung 1 .

(2) Je zwei der in den letzten $(m-1)$ Zeilen stehenden $n-T u p e l$ sind verschieden. Die resultierende Abbildung von $\{0,1\}^{X_{1}}$ in $\{0,1\}$ ist denn gerade die charakteristische Abbildung der Menge der n-Tupel, welche in den letzten (m-1) Zeilen stehen. Jede solche Menge ist eine (m-1)-elementige Teilmenge der Menge der $n-T u p e l$; von diesen Teilmengen gibt es $\left(\begin{array}{c}2^{n} \\ m-1\end{array}\right)$ verschiedene.

Es gilt nun also:

und somit:

$$
\left(\begin{array}{c}
2^{n} \\
m-1
\end{array}\right)<1+\left(\begin{array}{c}
2^{n} \\
m-1
\end{array}\right)=e_{i} \quad(1 \leq i \leq m)
$$

$$
\frac{1}{\log 5} \cdot m \cdot \log \left(\begin{array}{l}
2^{n} \\
m-1
\end{array}\right)<\ell(\varphi, v)
$$

Wird ausserdem $f$ durch $\varphi$ in minimaler Länge dargestellt, so gelten für die Länge von $\varphi$ die folgenden Schranken:

$$
\frac{1}{\log 5} \cdot m \cdot \log \left(\begin{array}{c}
2^{n} \\
m-1
\end{array}\right)<\ell(\varphi, v)<m^{2} \cdot n .
$$

Nun soll gezeigt werden, dass für spezielle Werte von $n$ und $m$ die bei- 
den Schranken von der gleichen Grössenordnung sind; genauer, dass sie beide von der Grössenordnung $\frac{(m \cdot n)^{2}}{\log (m \cdot n)}$ sind. Dabei ist $(m \cdot n)$ gerade die Anzahl der Variablen aus $V$.

Es sei $n$ positiv gerade, und $m=2^{n / 2}+1$.

Lemma 1 Für solche Werte von $n$ und $m$ gelten die Ungleichungen

$$
\begin{aligned}
& \frac{2}{3} \cdot \log (m \cdot n)<n<2 \cdot \log (m \cdot n) \\
& \frac{1}{3} \cdot \frac{(m \cdot n)}{\log (m \cdot n)}<2^{n / 2}
\end{aligned}
$$

Lemma 2 Für alle natürlichen Zahlen $r$ grösser als 1 gilt

$$
\frac{1}{6} \cdot r \cdot \log r<\frac{1}{\log 5} \cdot \log \left(r^{2} r\right)
$$

(Beweis mittels Abschätzung von Integralen.)

Aus diesen Lemmata folgt einerseits, dass

$$
\begin{aligned}
\frac{1}{36 \cdot \frac{(m \cdot n)^{2}}{\log (m \cdot n)}} & <\frac{1}{12} m \cdot n \cdot 2^{n / 2} \\
& =\frac{1}{6} \cdot m \cdot 2^{n / 2} \cdot \log 2^{n / 2} \\
& <\frac{1}{\log 5} \cdot m \cdot \log \left(\begin{array}{c}
2^{n} \\
2^{n / 2}
\end{array}\right) \\
& =\frac{1}{\log 5} \cdot m \cdot \log \left(\begin{array}{c}
2^{n} \\
m-1
\end{array}\right) ;
\end{aligned}
$$

und anderseits, dass

$$
m^{2} \cdot n<\frac{3}{2} \cdot \frac{(m \cdot n)^{2}}{\log (m \cdot n)}
$$

Ist also $\varphi$ eine Formel aus $\Phi(V)$, welche $f$ in minimaler Länge darstellt, so gelten für die Länge von $\varphi$ die folgenden Schranken:

$$
\frac{1}{36} \cdot \frac{(m \cdot n)^{2}}{\log (m \cdot n)}<2(\varphi, v)<\frac{3}{2} \cdot \frac{(m \cdot n)^{2}}{\log (m \cdot n)},
$$

falls $n$ positiv gerade ist, und $m=2^{n / 2}+1$; dabei ist $(m \cdot n)$ die Anzahl der Variablen aus $V$.

\section{Zweite Anwendung (Heiratsproblem)}

Es sei m eine natüriche Zahl grösser $31 s 3$, und $V$ die Menge der Variablen $x_{i, k}$ mit $1 \leq i, k \leq m$. Wir definieren eine Abbildung $f$ von $\left.\{0,1\}\right\}^{V}$ in $\{0,1\}$ : 
Ist $b$ eine Abbildung von $V$ in $\{0,1\}$, so ist genau dann $f(b)=1$, wenn es eine Permutation $p$ auf $\{1,2, \ldots, m\}$ gibt, sodass für alle Zahlen $i$ mit $1 \leq i \leq m$ gilt: $b\left(x_{i, p(i)}\right)=1$.

Ist $\varphi$ eine Fornel aus $\Phi(V)$, welche f darstellt, so besagt $\varphi$ in anschaulicher Ausdrucksweise, dass es in der Matrix

$$
\left(\begin{array}{ccc}
x_{1,1} & \cdots & x_{1, m} \\
\vdots & & \vdots \\
x_{m, 1} & \cdots & x_{m, m}
\end{array}\right)
$$

einen Weg der Länge $m$ gibt. Ein Weg der Länge s ist eine Menge von s Stellen $x_{i, p(i)}$, welche alle mit 1 belegt sind (dabei ist p eine Permutation auf $\{1, \ldots, m\})$.

Korollar 2 Für die Länge einer Formel $\varphi$, welche $f$ möglichst kurz darstellt, gilt:

$$
\frac{1}{32 \cdot \log 5} \cdot m^{3}<\ell(\varphi, v)<m^{2 m} \text {. }
$$

Zur oberen Schranke: Für jede Permutation $p$ auf $\{1, \ldots, m\}$ sei $\alpha_{p}$ die Formel

$$
\prod_{i=1}^{m} x_{i, p(i)}
$$

und $\alpha$ die Formel

$$
1+\prod_{p}\left(1+\alpha_{p}\right)
$$

Offenbar stellt $\alpha$ die Abbildung $f$ der. Bezüglich der Anzahl $n$ der Elemente von $V\left(n=m^{2}\right)$ gilt dann für die Länge von $\alpha$ :

$$
\ell(\alpha, v)=m \cdot m !<m^{2 m}=n^{\sqrt{n}} \text {. }
$$

Zur unteren Schranke: Wir wenden den Satz von Netschiporuk nicht auf $f$ an, sondern auf die folgende Hilfsabbildung $g$ von $\{0,1\}^{V}$ in $\{0,1\}$ : 2r sei die grösste gerade Zahl, welche kleiner als $m$ ist. Ist b eine Abbildung von $V$ in $\{0,1\}$, so ist genau dann $g(b)=1$, wenn es eine Permutation $p$ auf $\{1, \ldots, m\}$ gibt, sodass für mindestens $(2 r-1)$ verschiedene Zahlen $i(1 \leq i \leq 2 r)$ gilt: $b\left(x_{i, p}(i)\right)=1$.

Ist $\psi$ eine formel aus $\Phi(V)$, welche g darstellt, so besagt $\psi$ in anschaulicher Ausdrucksweise, dass es in der Matrix 


$$
\left(\begin{array}{ccc}
x_{1,1} & \cdots & x_{1,2 r} \\
\vdots & & \vdots \\
x_{2 r, 1} & x_{2 r, 2 r}
\end{array}\right)
$$

einen Weg der Länge $(2 r-4)$ oder $2 r$ gibt.

Für $1 \leq j \leq 2 r$ sei $x_{j}$ die Menge der Variablen $x_{i, k}$ mit $j+i=k+1$ (modulo $2 r$ ). D.h. es sei zum Beispiel für $r=2$ :

$$
\begin{aligned}
& x_{1}=\left\{x_{1,1}, x_{2}, 2, x_{3}, 3, x_{4,4}\right\}, \\
& x_{2}=\left\{x_{1,2}, x_{2,3}, x_{3,4}, x_{4,1}\right\}, \\
& x_{3}=\left\{x_{1,3}, x_{2,4}, x_{3,1}, x_{4,2}\right\}, \\
& x_{4}=\left\{x_{1,4}, x_{2,1}, x_{3,2}, x_{4,3}\right\} .
\end{aligned}
$$

$\left\{x_{1}, \ldots, x_{2 s}\right\}$ ist also eine Menge von disjunkten Teilmengen von $V$. Zur Abschätzung der Anzahl $e_{1}$ der Elemente von $E\left(g, X_{1}\right)$ zeigen wir folgendes:

Sind $c_{1}$ und $c_{2}$ zwei verschiedene Abbildungen von $V \times x_{1}$ in $\{0,1\}$, für welche gilt

$$
c_{1}\left(x_{i, k}\right)=c_{2}\left(x_{i, k}\right)=0, \text { falls } r<i \text { oder } k \leq r \text {, }
$$

so entsprechen $c_{1}$ und $c_{2}$ verschiedene Abbildungen auf $x_{1} ; d . h$. es gibt eine Abbildung $b$ von $x_{1}$ in $\{0,1\}$, sodass $f\left(\left[b, c_{1}\right]\right) \neq f\left(\left[b, c_{2}\right]\right)$.

Zum Beweis zeigen wir:

Ist $c_{1}\left(x_{i, k}\right)+c_{2}\left(x_{i, k}\right)$ und ist b definiert durch

$$
\begin{aligned}
& b\left(x_{j, j}\right)=1, \text { für } j \neq i, k, \\
& b\left(x_{i, i}\right)=b\left(x_{k, k}\right)=0,
\end{aligned}
$$

so ist $f\left(\left[b, c_{1}\right]\right) \neq f\left(\left[b, c_{2}\right]\right)$.

Dafür muss man offenbar beweisen, dass es in der Matrix

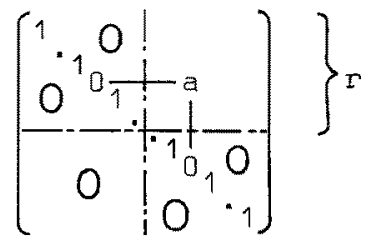


genau dann einen Weg der Länge (2r-1) oder $2 r$ gibt, wenn a gleich 1 ist. Falls a $=1$, gibt es ersichtlicher Weise einen solchen Weg.

Falls es umgekehrt einen solchen Weg gibt, so gehören alle $(2 r-2)$ Stellen der Diagonale dazu, welche durch b mit 1 belegt werden. (Fehlt z.B. eine der stellen in den ersten r Spalten, so enthält der Weg in diesen Spalten höchstens ( $r-2)$ Stellen, im Ganzen also höchstens (2r-2) Stellen.) Ausser den Stellen $x_{j, j}(j \neq i, k)$ kann ein solcher Weg höchstens noch die Stellen $x_{i, i}, x_{i, k}, x_{k}, i, x_{k}, k$ enthalten. Nach Konstruktion ist nun $b\left(x_{i, i}\right)=b\left(x_{k, i}\right)=b\left(x_{k, k}\right)=0$. Also muss der Weg die Stelle $x_{i, k}$ enthalten, und d.h., dass $a=1$.

Da es $2^{\left(r^{2}\right)}$ verschiedene Abbildungen von $W_{X}$ in $\{0,1\}$ mit der oben genannten Eigenschaft gibt, ist damit also gezeigt, dass $r^{2} \leq \log e_{1}$. Offensichtich ist $e_{1}=e_{2}=\ldots=e_{2 r}$.

Daher gilt nach dem Satz von Netschiporuk für die Länge einer Formel $\psi$ aus $\Phi(V)$, welche $g$ darstellt:

$$
\frac{1}{\log 5} \cdot \sum_{j=1}^{2 r} r^{2}=\frac{2}{\log 5} \cdot r^{3}<\ell(\psi, v) .
$$

Es sei $\varphi$ eine Formel aus $\Phi(V)$, welche die eingangs definierte Abbildung f darstellt.

Ist $m=2 r+1$, so stellt die Formel $\varphi^{\prime}$, welche durch Substitution von 1 für $x_{i, m} x_{m, i}(1 \leq i \leq m)$ aus $\varphi$ entsteht, die Hilfsabbildung g dar. Anschaulich gesprochen gibt es nämlich genau dann in der Matrix

$$
\left(\begin{array}{cccc}
x_{1,1} & \cdots & x_{1,2 r} & 1 \\
\vdots & & \vdots & \vdots \\
x_{2 r, 1} & \cdots & x_{2 r, 2 r} & 1 \\
1 & \cdots & 1 & 1
\end{array}\right)
$$

einen Weg der Länge $(2 r+1)$, wenn es in der Matrix

$$
\left(\begin{array}{ccc}
x_{1,1} & \cdots & x_{1,2 r} \\
\vdots & & \vdots \\
x_{2 r, 1} & \cdots & x_{2 r, 2 r}
\end{array}\right)
$$

einen Weg der Länge $\left(2 r^{-1}\right)$ oder $2 r$ gibt. 
Offenbar ist

$$
\frac{2}{\log 5} \cdot r^{3}<\ell\left(\varphi^{\prime}, V\right) \leq \ell(\varphi, V) .
$$

Ist $m=2 r+2$, so stellt die Formel $\varphi^{\prime \prime}$, welche durch Substitution von 1 für $x_{m, m}, x_{m-1, i}, x_{i}, m-1,(1 \leq i \leq m-1)$ und durch substitution von 0 für $x_{m, j}, x_{j, m}(1 \leq j \leq m-1)$ aus $\varphi$ entsteht, die Hilfsfunktion $g$ dar. Anschaulich gesprochen gibt es nämlich genau dann in der Matrix

$$
\left(\begin{array}{ccccc}
x_{1,1} & \cdots & x_{1,2 r} & 1 & 0 \\
\vdots & & \vdots & \vdots & \vdots \\
x_{2 r, 1} & \cdots & x_{2 r, 2 r} & 1 & 0 \\
1 & \cdots & 1 & 1 & 0 \\
0 & \cdots & 0 & 0 & 1
\end{array}\right)
$$

einen Weg der Länge $(2 r+2)$, wenn es in der Matrix

$$
\left(\begin{array}{ccc}
x_{1,1} & \cdots & x_{1,2 r} \\
\vdots & & \vdots \\
x_{2 r, 1} & \cdots & x_{2 r, 2 r}
\end{array}\right) .
$$

einen Weg der Länge $\left(2 r^{-1}\right)$ oder 25 gibt.

offenbar ist

$$
\frac{2}{\log 5} \cdot r^{3}<\ell\left(\varphi^{\prime \prime}, V\right) \leq \ell(\varphi, V)
$$

Da $3 \leq m$, gilt aber in beiden Fälen: $\frac{m}{4} \leq r$, und somit:

$$
\frac{1}{32 \cdot \log 5} \cdot m^{3}<\ell(\varphi, V) \text {. }
$$

Stellt nun $\varphi$ die Abbildung $f$ von $\{0,1\}^{V}$ in $\{0,1\}$ ausserdem in minimaler Länge dar, so gelten bezüglich der Anzahl $n$ der Elemente von $V\left(n=m^{2}\right)$ für die Länge von $\varphi$ die folgenden Abschätzungen:

$$
\frac{1}{32 \cdot \log 5} \cdot n^{3 / 2}<\ell(\varphi, v)<n^{\sqrt{n}} .
$$




\section{Dritte Anwendung}

Der Satz von Netschiporuk soll nun angewandt werden auf Formeln, welche symmetrische Abbildungen darstellen. Wir zeigen, dass der Satz für die Länge solcher Formeln nur triviale untere Schranken liefert.

Es sei $n$ eine natürliche Zahl, und $V$ die Menge der Variablen $x_{i}$ mit $1 \leq i \leq n$. Für jede Teilmenge $Q$ von $\{a, 1, \ldots, n\}$ definieren wir eine Abbildung $f_{Q}$ von $\{0,1\}^{V}$ in $\{0,1\}$ :

Ist b eine Abbildung von $V$ in $\{0,1\}$, und $r_{b}$ die Anzahl der natürlichen Zahlen $j$ mit $1 \leq j \leq n$ und $b\left(x_{j}\right)=1$, so ist genau dann $f_{Q}(b)=1$, wenn $r_{b}$ in $Q$.

Es gibt genau $2^{(n+1)}$ verschiedene solche Abbildungen $f_{Q}$, und diese sind gerade die symmetrischen Abbildungen von $\{0,1\}^{V}$ in $\{0,1\}$.

Ist zum Beispiel $2 \leq n$, und $Q=\{2, \ldots, n\}$, so wird $f_{Q}$ dargestellt durch die Formel

$$
1+\Pi\left(1+x_{i} \cdot x_{j}\right) \quad(1 \leq i<j \leq n)
$$

Es sei $\left\{x_{1}, \ldots, x_{m}\right\}$ eine Menge von disjunkten Teilmengen von $V$. Es sei zudem $n_{i}$ die Anzahl der Elemente von $X_{i}$, und $e_{i}$ die Anzahl der Elemente von $E\left(f_{Q}, X_{i}\right)$. In $E\left(f_{Q}, X_{i}\right)$ sind aber nur symmetrische Abbildungen enthalten. Darum ist $e_{i} \leq 2\left(n_{i}+1\right)$.

Setzt man ausserdem voraus, dass $2<e_{i}(1 \leq i \leq m)$, so besagt der Satz von Netschiporuk folgendes:

$$
\frac{1}{\log 5} \cdot \sum_{i=1}^{m} \log e_{i}<\ell(\varphi, v),
$$

falls $\varphi$ eine Formel aus $\Phi(V)$ ist, welche $f_{Q}$ darstellt.

Es ist aber $\left(n_{1}+\ldots+n_{m}\right) \leq n$, und unter diesen speziellen Voraussetzungen $m \leq n$. Somit gilt:

$$
\begin{aligned}
\frac{1}{\log 5} \cdot \sum_{i=1}^{m} \log e_{i} & \leq \frac{1}{\log 5} \cdot \sum_{i=1}^{m}\left(n_{i}+1\right) \\
& \leq \frac{1}{\log 5} \cdot(n+m) \\
& \leq \frac{2}{\log 5} \cdot n \\
& <n .
\end{aligned}
$$


Der Satz von Netschiporuk besagt also für symmetrische Abbildungen von $\{0,1\}^{V}$ in $\{0,1\}$ nie mehr, als dass deren Darstellungen Längen haben, die mindestens so gross sind wie die Anzahl der Elemente von $V$ - d.h. wie die Anzahl der Variablen.

\section{Verallgemeinerung}

Es soll hier gezeigt werden, dass man mit den gleichen Methoden, wie sie im Beweis des Lemmas auf Seite 4 benützt wurden, auch den allgemeinen Satz von Netschiporuk für Formeln über beliebigen Basen beweisen kann.

Es sei $V$ eine Menge von Variablen, q eine natürliche Zahl grösser als 1. und $B_{q}$ die Menge der Zeichen für die Abbildungen von $\{0,1\}^{q}$ in $\{0,1\}$ mit genau q wesentlichen Argumentstellen. $\Phi_{q}(V)$ sei die kleinste Menge $F$ von Formeln, welche die folgenden Eigenschaften hat: Die Formeln 0,1 und die Variablen aus $V$ gehören $z u F$; ist b aus $B_{q}$, so gehört mit $\varphi_{1}, \varphi_{2}, \ldots, \varphi_{q}$ auch $b\left(\varphi_{1}, \ldots, \varphi_{q}\right) z u F$.

Für eine Formel $\varphi$ aus $\Phi_{q}(V)$ und eine Teilmenge $X$ von $V$ sei $\ell(\varphi, X)$ die mit Vielfachheit gezählte - AnzahI der Variablen aus $X$ in $\varphi$.

Einer Formel $\varphi$ aus $\Phi_{q}(V)$ ist in bekannter Weise eine Abbildung $\hat{\varphi}$ von $\{0,1\}^{V}$ in $\{0,1\}$ zugeordnet.

Für eine Formel $\varphi$ aus $\Phi_{q}(V)$ und eine Teilmenge $X$ von $V$ seien $E(\hat{\varphi}, X)$ und $\bar{E}(\hat{\varphi}, X)$ die Mengen von Abbildungen, wie sie im Spezialfall definiert wurden. Offenbar ist $E(\hat{\varphi}, X) \subset \bar{E}(\hat{\varphi}, X)$.

Lemma Es sei $\varphi$ eine Formel aus $\Phi_{q}(V), X$ eine Teilmenge von $V$. Dann ist die Anzahl a der Elemente van $\bar{E}(\hat{\varphi}, X)$ höchstens gleich

$$
2^{(1-q)} \cdot\left(\left(2^{q}+1\right)^{\ell(\varphi, x)}+\left(2^{q}-1\right)\right) \text {. }
$$

Wir beweisen das mit Induktion nach dem Formelaufbau:

Ist $\varphi$ eine der Formeln 0,1 , oder eine Variable, welche nicht $z u X$ gehört, so ist $\ell(\varphi, X)=0$, und $\bar{E}(\hat{\varphi}, X)$ besteht aus den beiden konstanten Abbildungen; es ist aber $2^{(1-q)} \cdot\left(\left(2^{q}+1\right)^{0}+\left(2^{q}-1\right)\right)=2$.

Ist $\varphi$ eine Variable aus $X$, so ist $\ell(\varphi, X)=1$, und $\bar{E}(\hat{\varphi}, X)$ besteht aus 4 Abbildungen; es ist aber $2^{(1-q)} \cdot\left(\left(2^{q}+1\right)^{1}+\left(2^{q}-1\right)\right)=4$.

Es sei nun $\varphi$ die Formel $b\left(\varphi_{1}, \ldots, \varphi_{q}\right)$, wo b aus $B_{q}$ ist. Es ist dann $\ell(\varphi, X)=\ell\left(\varphi_{1}, X\right)+\ldots+\ell\left(\varphi_{q}, X\right)$. Ist $f$ ein Element von $\bar{E}(\bar{\varphi}, X)$, so ist $f$ 
konstant, oder es gibt Elemente $f_{i}$ in $\bar{E}\left(\hat{\varphi}_{i}, X\right)$ und eine Konstante $c$, sodass $f=\hat{b}\left(f_{1}, \ldots, f_{q}\right)+c$. (Dabei deutet + die Boole'sche Summe, und $\hat{b}$ die Boole'sche Abbildung zum Zeichen b an.)

Die Anzahl der Elemente von $\bar{E}\left(\hat{\varphi}_{i}, x\right)$ sei gleich $a_{i}$. Zur Abschätzung der Anzahl a der Elemente von $\bar{E}(\hat{\varphi}, X)$ unterscheiden wir 3 Fälle:

(1) $f$ ist konstant, oder $f=\hat{b}\left(f_{1}, \ldots, f_{q}\right)+c$, wobei alle $f_{i}$ konstant sind.

In diesem Fall ist $f$ konstant; es gibt zwei konstante Abbildungen.

(2) Es ist $f=\vec{b}\left(f_{1}, \ldots, f_{q}\right)+c$, und es gibt ein $i(1 \leq i \leq q)$ mit:

$f_{1}, \ldots, f_{i-1}, f_{i+1}, \ldots, f_{q}$ sind konstant, $f_{i}$ ist nicht konstant. Es gibt dann Konstanten $c$ und $d$, sodass $f=0 \cdot f_{i}+d$. Sol $f$ nicht

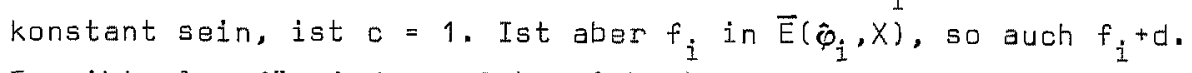
Es gibt also für jedes solches $i$ höchstens $a_{i}-2$, für diesen Fall insgesamt höchstens $\left(a_{1}-2\right)+\ldots+\left(a_{q}-2\right)$ nicht kanstante Elemente.

(3) Es ist $f=\hat{b}\left(f_{1}, \ldots, f_{q}\right)+c$, und es gibt eine k-elementige Teilmenge $I$ von $\{1, \ldots, q\} \quad(2 \leq k \leq q)$ mit: $f_{i}$ ist genau dann konstant, wenn $i$ nicht in $I$.

(Oie Menge der k-elementigen Teilmengen von $\{1, \ldots, q\}$ bezeichnen wir mit $P_{k} \cdot$ J

Für jedes konstante $f_{i}$ und für o gibt es höchstens 2, für jedes nicht konstante $f_{i}$ höchstens $a_{i}-2$ Möglichkeiten. Es werden also für jedes $I$ aus $P_{k}$ höchstens

$$
2 \cdot 2^{(q-k)} \cdot \prod_{i \in I}\left(a_{i}-2\right),
$$

und in diesem Fall insgesamt höchstens

$$
2 \cdot \sum_{k=2}^{q} \sum_{I \in P_{k}} 2^{(q-k)} \cdot \prod_{i \in I}\left(a_{i}-2\right)
$$

Abbildungen dargestellt.

Zusammenfassend erhalten wir die Abschätzung:

$$
\begin{aligned}
a & \leq 2+\sum_{i=1}^{q}\left(a_{i}-2\right)+2 \cdot \sum_{k=2}^{q} \sum_{I \in P_{k}} 2^{(q-k)} \cdot \prod_{i \in I}\left(a_{i}-2\right) \\
& =2 \cdot \prod_{i=1} a_{i}-\left(2^{q}-1\right) \cdot \sum_{i=1}^{q}\left(a_{i}-2\right)-2 \cdot\left(2^{q}-1\right) \\
& \left.\leq 2^{(1-q)} \cdot\left(\prod_{i=1}^{q} 2^{(q-1)} \cdot\left(a_{i}-2\right)+1\right)+\left(2^{q}-1\right)\right) .
\end{aligned}
$$

Zum Beweis der letzten Ungleichung $L \leq R$ zeigt man, dass für alle 
natürlichen Zahlen $q, a_{1}, \ldots a_{q}$, welche grösser oder gleich 2 sind, gilt:

$$
\begin{aligned}
(L-R)= & -2^{(1-q)} \cdot \prod_{i=1}^{q}\left(2^{(q-1)} \cdot a_{i}-2^{q}+1\right)+z \cdot \prod_{i=1}^{q} a_{i} \\
& -\left(2^{q}-1\right) \cdot \sum_{i=1}^{q}\left(a_{i}-2\right)-2^{(q+1)}+2^{(1-q)} \\
= & \sum_{i=1}^{q}\left(2-a_{i}\right) \cdot\left(\prod_{j=1}^{i-1}\left(2^{(q-1)} a_{j}-2^{q}+1\right)-2^{q} \cdot \prod_{j=1}^{i-1} a_{j} / 2+2^{q}-1\right) ;
\end{aligned}
$$

dieser letzte Ausdruck ist aber kleiner oder gleich 0 , weil bei jedem Glied der Summe jeweils der erste Faktor kleiner oder gleich 0 , und der zweite Faktor - wie man leicht mit Induktion nach $i(1 \leq i \leq q)$ zeigt grösser oder gleich 0 ist.

Nach Induktionsvoraussetzung ist

$$
2^{(q-1)}\left(a_{i}-2\right)+1 \leq\left(2^{q}+1\right)^{l\left(\varphi_{i}, X\right)} .
$$

Deshalb gilt für die Anzahl a der Elemente von $\bar{E}(\hat{\varphi}, X)$ :

$$
\begin{aligned}
a & \leq 2^{(1-q)} \cdot\left(\prod_{i=1}^{q}\left(2^{q}+1\right)^{l\left(\varphi_{i}, x\right)}+\left(2^{q}-1\right)\right) \\
& =2^{(1-q)} \cdot\left(\left(2^{q}+1\right)^{l(\varphi, x)}+\left(2^{q}-1\right)\right) .
\end{aligned}
$$

Wir werden das Lemma in der folgenden form verwenden:

Korollar Es sei $\varphi$ eine Formel aus $\Phi_{q}(V)$, und $X$ eine Teilmenge von $V$. Ist dann die Anzahl $n$ der Elemente von $E(\hat{\varphi}, X)$ grösser als 2 , so gilt für die Länge von $\varphi$ in Variablen aus $Y$ die folgende Abschätzung:

$$
\frac{\log n}{\log \left(2^{q}+1\right)}<\ell(\varphi, x) \text {. }
$$

Beweis Nach dem Lemma ist

$$
n \leq a \leq 2^{(1-q)} \cdot\left(\left(2^{q}+1\right)^{l(\varphi, x)}+\left(2^{q}-1\right)\right) .
$$

Für positive ganze Zahlen $m$ und $q(2 \leq q)$ gilt aber

$$
2^{(1-q)} \cdot\left(\left(2^{a}+1\right)^{m}+\left(2^{q}-1\right)\right)<\left(2^{q}+1\right)^{m} \text {; }
$$

und somit ist $\log n<\ell(\varphi, x) \cdot \log \left(2^{q}+1\right)$.

Satz Es sei $V$ eine Menge von Variablen, f eine Abbildung von $\{0,1\}$ in $\{0,1\}$. $\varphi$ eine Formel aus $\varphi_{q}(V)$, welche $f$ darstellt, und $\left\{x_{1}, \ldots, x_{m}\right\}$ eine Menge von disjunkten Teilmengen von $V$. Die Anzahl $e_{i}$ der Elemente 
von $E\left(f, X_{i}\right)$ sei grösser als $2(1 \leq i \leq m)$. Dann gilt

$$
\frac{1}{\log \left(2^{q}+1\right)} \cdot \sum_{i=1}^{m} \log e_{i}<\ell(\varphi, v) .
$$

Zum Beweis:Da die Mengen $x_{1}, \ldots, x_{m}$ disjunkt sind, ist

$$
\sum_{i=1}^{m} \ell\left(\varphi, x_{i}\right) \leq \ell(\varphi, v) \text {. }
$$

Bemerkung Ist $V$ eine Variablenmenge, und sind $p$, q ganze Zahlen mit $0 \leq p \leq q, 2 \leq q$, so kann jede Abbildung von $\{0,1\}^{V}$ in $\{0,1\}$ mit p Argumentstellen durch eine Formel $\varphi$ aus $\Phi_{q}(V)$ der Länge p dargestellt werden, d.h. $\ell(\varphi, V)=p$.

Deshalb gilt der Satz von Netschiporuk auch in der folgenden Form:

Satz Es sei $V$ eine Menge von Variablen, $f$ eine Abbildung von $\{0,1\}^{V}$ in $\{0,1\}$, und $\varphi$ eine Formel, die nur Zeichen von Abbildungen mit höchstens q Argumentstellen enthält. Ist dann $\left\{x_{1}, \ldots, x_{m}\right\}$ eine Menge von disjunkten Teilmengen von $V$, und die Anzahl $e_{i}$ der Elemente von $E\left(f, x_{i}\right)$ grösser als $2(1 \leq i \leq m)$, so gilt

$$
\frac{1}{\log \left(2^{q}+1\right)} \cdot \sum_{i=1}^{m} \log e_{i}<\ell(\varphi, v) .
$$

Folgerung Der Satz von Netschiporuk allein liefert niemals Funktionenfolgen, mit denen Basenvergleiche zu machen sind (Subbotovskaja 1963). Für $2 \leq q<q$ ' möchte man zum Beispiel zeigen, dass die Basen $B_{q}$ und $B_{q}$ ' nicht äquivalent sind. Bereits aus der Bemerkung oben weiss man, dass

$$
B_{q}, \leqslant B_{q} ;
$$

d.h.: Es gibt eine Konstante $M$, welche nur von $B_{q}$ und $B_{q}$, abhängt, sodess für jede Abbildung $f$ von $\{0,1\}^{V}$ in $\{0,1\}$ eine $f_{n}{ }^{q}$-darstellende Formel $\psi$ aus $\Phi_{q},(V)$ existiert,

sodass für jede $f_{n}$-darstellende Formel $\varphi$ aus $\Phi_{q}(V)$ gilt:

$$
\ell(\psi, V) \leq M \cdot \ell(\varphi, V) \text {. }
$$

Was man zeigen möchte, ich also, dass

$$
\operatorname{nicht} B_{q} \leqslant B_{q} \text {. }
$$

Angenommen $\left\{V_{n}\right\}$ sei eine Folge von Mengen von Variablen, und es sei $f_{n}:\{0,1\} \stackrel{V_{n}}{\Longrightarrow}\{0,1\}$ eine Folge von Abbildungen $(n=1,2, \ldots)$. Weiterhin angenommen aus dem Satz von Netschiporuk wäre das folgende Resultat vorhanden: 
Ist $\varphi$ eine Formel aus $\Phi_{q}\left(V_{n}\right)$, welche $f_{n}$ darstellt, so gilt

$$
\frac{1}{\log \left(2^{q}+1\right)} \cdot s_{n}<\ell\left(\varphi, v_{n}\right) \text {. }
$$

Dann könnte (*) bewiesen werden, indem man folgendes zeigt:

Zu jeder Konstanten $M$ gibt es eine Zahl $n$, sodass es zu jeder $f_{n}$-darstellenden Formel $\varphi$ aus $\Phi_{q}\left(V_{n}\right)$ eine $f_{n}$-darstellende Formel $\psi$ aus $\Phi_{q},\left(V_{n}\right)$ gibt, wofür gilt

$$
M \cdot \ell\left(\psi, v_{n}\right) \leq \frac{1}{\log \left(2^{q}+1\right)} \cdot S_{n}<\ell\left(\varphi, v_{n}\right) .
$$

Nun liefert aber der Satz von Netschiporuk auch, dass

$$
\frac{1}{\log \left(2^{q^{\prime}}+1\right)} \cdot s_{n}<\ell\left(\psi, v_{n}\right)
$$

und somit

$$
\frac{1}{\log \left(2^{a}+1\right)} \cdot S_{n}<\frac{\log \left(2^{q^{\prime}}+1\right)}{\log \left(2^{a}+1\right)} \cdot 2\left(\psi \cdot v_{n}\right)
$$

Damit aber wird der gewünschte Schluss verunmöglicht.

\section{Teil}

Es sei $V$ eine Menge von Variablen, und $K$ eine Menge von Konstanten, welche 0 und 1 enthält. $\Phi(V, K)$ sei die kleinste Menge F von Formeln, welche die folgenden Eigenschaften hat: Die Variablen aus $V$ und die Konstanten aus $K$ gehären zu F; mit $\varphi$ und $\psi$ gehören auch $(\varphi+\psi)$ und $(\varphi \cdot \psi)$ zu F. Für $\Phi(\{v\}, K)$ schreiben wir kürzer $\Phi(v, K)$.

Ist $\hat{k}$ ein endlicher kommutativer Ring mit Einselement und mit dem Universum $K$, so ist in bekannter Weise jeder Formel $\varphi$ aus $\Phi(V, K)$ eine $A b-$ bildung $\hat{\varphi}(\hat{k})$ von $K^{V}$ in $K$ zugeordnet.

Es seien $\varphi$ und $\psi$ Formeln aus $\Phi(V, K), X$ eine Teilmenge und $v$ ein Element von $V$. Denn definieren wir:

$\ell(\varphi, X)$ sei die - mit Vielfachheit gezählte - Anzahl der Variablen aus $X$ in $\varphi . \ell(\varphi, V)$ ist die Länge von $\varphi$. Für $\ell(\varphi,\{v\})$ schreiben wir kürzer $\ell(\varphi, v)$.

$h(\varphi, x)$ sei das Maximum von $\ell(\varphi, x)$ für $x$ aus $x$.

Var $\varphi$ sei die Menge der Variablen aus $V$, welche in $\varphi$ vorkommen. Für $\operatorname{Var} \varphi_{1} \cup \ldots$ V Var $\varphi_{n}$ schreiben wir kürzer $\operatorname{Var}\left(\varphi_{1} \cdot \ldots \cdot \varphi_{n}\right)$. 
$\varphi / X$ sei diejenige Formel, welche aus $\varphi$ entsteht, wenn alle Variablen aus $V \backslash X$ überall durch 0 ersetzt werden.

$\left.\varphi\right|_{\psi} ^{\vee}$ sei diejenige Formel, welche aus $\varphi$ entsteht, wenn die Variable $v$ überall durch $\psi$ ersetzt wird.

Bezugnehmend auf eine Interpretation der Formeln aus $\Phi(V, K)$ in einem endichen kommutativen Ring $k$ mit Einselement, definieren wir für Formeln $\varphi$ und $\psi$ aus $\Phi(V, K)$ :

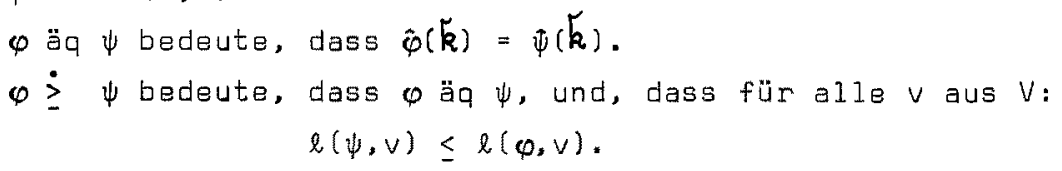

Bemerkung Für Formeln $\varphi$ und $\psi$ aus $\Phi(V, K)$ und eine Teilmenge $x$ von $V$ gilt:

$$
\begin{aligned}
& h(\varphi / X, V) \leq h(\varphi, V) . \\
& \text { Ist } \varphi \gtreqless \psi, \text { so ist } h(\psi, V) \leq h(\varphi, V) .
\end{aligned}
$$

Formeln aus $\Phi(V, K)$ von der form

$$
\left.(\alpha)_{\varphi}^{x}+\left(\left.B\right|_{\varphi} ^{x}=\psi\right)\right) \text {, }
$$

wo $\alpha$ und $\beta$ aus $\Phi(x, K)$ (d.h. Formeln in einer Variablen !), $\varphi$ und $\psi$ aus $\Phi(V, K)$, bezeichnen wir mit $\left(\varphi^{*} \psi\right)$.

Jedes Paar $\langle\alpha, \beta\rangle$ aus $\Phi(x, k)^{2}$ definiert also eine Sternoperation. Zur Unterscheidung von Sternoperationen werden die Sterne indiziert, apostrophiert und überstrichen.

Bemerkung Werden die Formeln im Körper $k_{0}$, dem Körper mit dem Universum $\{0,1\}$ interpretiert, gibt es zu jeder forme $l \varphi$ aus $\bar{\varphi}(\{x, y\}, K)$ eine Sternoperation *, sodass $\varphi$ äq $(x * y)$.

Bemerkung Zu Formeln $\varphi$ und $\psi$ aus $\Phi(V, K)$, einer Variablen $V$ aus $V$, einer Konstanten c aus $K$, und Sternoperationen ${ }_{1}^{*}$ und ${ }_{2}^{*}$ gibt es Sternoperationen ${ }_{3}^{*}, \stackrel{*}{4}$ und $\stackrel{*}{5}$ mit:

$$
\begin{aligned}
& \left(v_{1}^{*}\left(v_{2}^{*} \varphi\right)\right) \text { äq }\left(v_{3}^{*} \varphi\right) . \\
& \left(c_{1}^{*}\left(\varphi_{2}^{*} \psi\right)\right) \doteq\left(\varphi_{4}^{*} \psi\right) . \\
& \text { Ist var } \varphi=\{v\}, \text { so ist }\left(\varphi_{1}^{*} \psi\right) \gtreqless\left(v_{5}^{*} \psi\right) .
\end{aligned}
$$

Ausserdem zeigt man leicht (mit Induktion nach dem Aufbau von $\varphi$ !), dass es zu jedern $\varphi$ aus $\Phi(V, K)$ und jeder Teilmenge $X$ von $V$ Formein $X$ und $w$ aus $\Phi(V, K)$ und eine Sternoperation * gibt mit: 


$$
\begin{aligned}
\varphi & \geq\left(x^{*} \omega\right) . \\
1 & \leq|\operatorname{Var} x| . \\
\frac{1}{2} & \cdot|\operatorname{Var} \varphi \backslash x| \leq|\operatorname{Var} \omega \backslash x| .
\end{aligned}
$$

Zur einfacheren Formulierung des folgenden Lemma 1 definieren wir eine zahlentheoretische Abbildung:

$$
\begin{aligned}
& I: \quad\{1,2, \ldots\}^{4} \Longrightarrow\{1,2, \ldots\} ; \\
& \Gamma(k, m, q, r)=4^{(k+1)^{k \cdot r}-1} \cdot q+m .
\end{aligned}
$$

\section{Lemma 1}

Zu jedem endlichen kommutativen Ring $k$ mit Einselement und mit dem Universum $K$,

zu jeder Variablenmenge $V$ und allen natürlichen Zahlen $k, m, q, r$ mit $\Gamma(k, m, q, r) \leq|V|$,

und zu jeder Formel $\varphi$ aus $\Phi(V, K)$ mit $h(\varphi, V) \leq k$, gibt es eine Teilmenge $W$ von $V$, für welche einer der drei folgenden Fälle zutrifft:

(I) $\varphi / W$ ist äquivalent einer Konstanten aus $K$, und $|W|=m$;

(II) es gibt Formeln $\rho, \sigma$ und eine Sternoperation * mit:

$\varphi / W \geq(\rho * \sigma)$, und

$q \leq \mid$ Varo $\cap$ Varo $\mid$;

(III) $|W|=\Gamma$, und

es gibt eine Formel $\omega$ und Sternoperationen $\underset{1}{\bar{*}}, \ldots, \underset{\mathrm{F}}{\bar{*}}$ mit:

$\varphi / W$ äq $\left(w_{1}{ }_{1}^{\bar{x}} \ldots\left(w_{r} \underset{n}{*} \omega\right) \ldots\right)$

wo $\left\{w_{1}, w_{2}, \ldots, w_{n}\right\}=w$, und $h(w, v) \leq(k-1)$.

\section{Beweis}

Falis $m \leq \mid$ VVar $\varphi \mid$, trifft für $W=$ WVar $\varphi$ der Fall (I) $z u$. Andern-

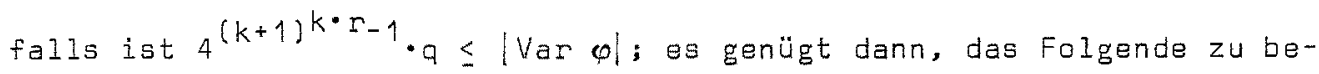
weisen:

Wenn es keine Teilmenge $W$ van $V$ gibt, sodass (II), so gibt es eine Teilmenge $w$ von $V$, sodass (III).

1. Schritt Es sei $p=(k+1)^{(k \cdot r)}$. Wir definieren rekursiv eine Folge von Formeln aus $\Phi(V, K)$ : 


$$
\begin{aligned}
& \left(\psi_{0} \quad \begin{array}{ll}
* & \varphi_{1}
\end{array}\right) \\
& \left.\left(\begin{array}{llll}
\psi_{0} & \stackrel{*}{*}\left(\psi_{1}\right. & 2 & \varphi_{2}
\end{array}\right)\right) \\
& \left(\psi_{0}{ }_{1}^{*}\left(\psi_{1} 2_{2}^{*} \ldots\left(\psi_{i-1}{ }_{i}^{*} \varphi_{i}\right) \ldots\right)\right) \\
& \psi_{0}^{*}\left(\psi_{1} 2_{2}^{*} \ldots\left(\psi_{p-1} \stackrel{p}{p}_{p}\right) \ldots\right)
\end{aligned}
$$

Mit den Bezeichnungen

$$
\begin{aligned}
& x_{i}=\operatorname{Var}\left(\psi_{0} \cdot \ldots \cdot \psi_{i-1} \cdot \varphi_{i}\right) \\
& y_{i-1}=\operatorname{var}\left(\psi_{0} \cdot \ldots \cdot \psi_{i-1}\right)
\end{aligned}
$$

soll für diese Formelfolge gelten:

(a) für alle $i \quad(1 \leq i \leq p)$ :

$$
\begin{aligned}
& \varphi / x_{i} \geq\left(\psi_{0}{ }_{1}^{*} \ldots\left(\psi_{i-1} \stackrel{i}{i}_{i}\right) \ldots\right) \text {, und } \\
& 4^{(p-i)} \cdot q \leq\left|X_{i} \backslash Y_{i-1}\right|=\left|\operatorname{Var} \varphi_{i} \backslash Y_{i-1}\right|
\end{aligned}
$$

(b) für alle i $(2 \leq i \leq p)$ :

$$
\begin{aligned}
& \left|\operatorname{Var} \psi_{i-1}\right|=1 \text {, oder } \\
& \left|\operatorname{Var} \psi_{i-1}\right|>1 \text { und } \operatorname{Var} \psi_{i-1} \subset Y_{i-2} .
\end{aligned}
$$

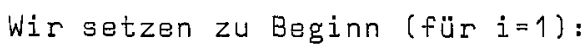

$$
\begin{aligned}
& \psi_{0} \text { sei } a, \varphi_{1} \text { sei } \varphi \text {, und } \\
& \left(\psi_{0}{ }_{1}^{*} \varphi_{1}\right) \text { sei }\left(\psi_{0}+\left(\left(1+\psi_{0}\right) \cdot \varphi_{1}\right)\right) .
\end{aligned}
$$

Da $X_{1}=X_{1} \backslash Y_{0}=\operatorname{Var} \varphi$, ist (a) sicher erfüllt.

Wir nehmen nun an, die Formelfolge sei für irgendein $i(1 \leq i \leq p-1)$ definiert bis $\left(\psi_{0}{ }_{1}^{*} \ldots\left(\psi_{i-1} i_{i}^{*} \varphi_{i}\right) \ldots\right)$. Nach unserer Bemerkung über Sternoperationen auf $S .20$ gibt es nun aber Formeln $x_{i}$ und $\omega_{i}$, und eine Sternoperation * mit:

$$
\begin{aligned}
& \varphi_{i} \geq\left(x_{i} * \omega_{i}\right) . \\
& 1 \leq\left|\operatorname{Var} x_{i}\right| . \\
& \frac{1}{2} \cdot\left|\operatorname{Var} \varphi_{i} \backslash Y_{i-1}\right| \leq\left|\operatorname{Var} \omega_{i} \backslash Y_{i-1}\right| .
\end{aligned}
$$

Wir bezeichnen einige, parweise disjunkte, Teilmengen von $x_{i}$ :

$$
\begin{aligned}
& A=\left(\operatorname{Var} \chi_{i} \cap \operatorname{Var} \omega_{i}\right) \backslash Y_{i-1} \\
& B=\left(\operatorname{Var} \chi_{i} \cap \operatorname{Var} \omega_{i}\right) \cap Y_{i-1} \\
& C=\left(\operatorname{Var} \omega_{i} \backslash \operatorname{Var} \chi_{i}\right) \backslash Y_{i-1} \\
& D=\left(\operatorname{Var} \omega_{i} \backslash \operatorname{Var} \chi_{i}\right) \cap Y_{i-1}
\end{aligned}
$$




$$
\begin{aligned}
& E=\left(\operatorname{Var} x_{i} \backslash \operatorname{Var} \omega_{i}\right) \backslash y_{i-1} \\
& F=\left(\operatorname{Var} \chi_{i} \backslash \operatorname{Var} \omega_{i}\right) \cap Y_{i-1}
\end{aligned}
$$

Es sei $\operatorname{U} \cup F \neq \varnothing$ :

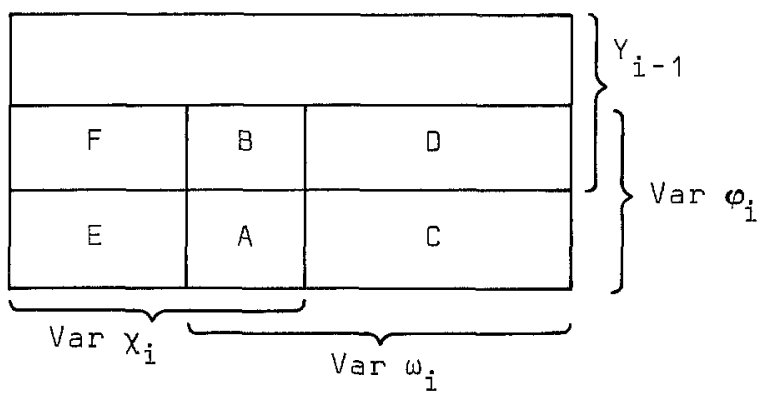

In diesem Fall setzen wir:

$$
\begin{aligned}
& \psi_{i} \operatorname{sei} x_{i} /(B \cup F), \\
& \varphi_{i+1} \operatorname{sei} \omega_{i} /(B \cup D \cup C) .
\end{aligned}
$$

Die Bedingung (b) ist dann sicher erfüllt, weil

$$
\begin{aligned}
& Y_{i}=Y_{i-1}, \\
& X_{i+1}=Y_{i} \cup C, \\
& \operatorname{Var} \psi_{i} \subset Y_{i-1} .
\end{aligned}
$$

Es sei $B \cup F=\emptyset$, und $x$ aus $\operatorname{Var} x_{i}$ :

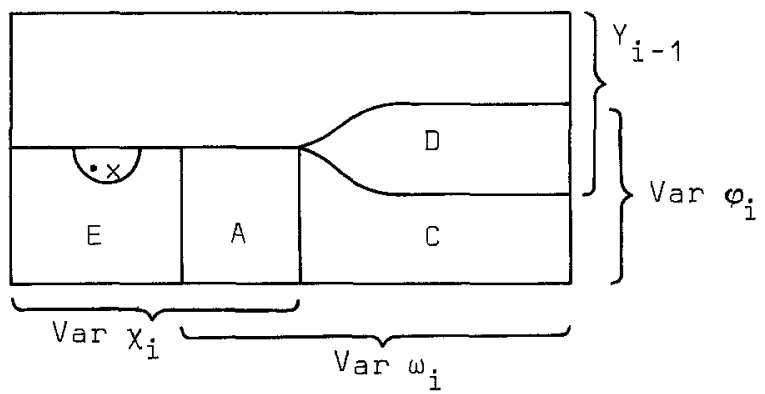

In diesem Fall setzen wir:

$$
\begin{aligned}
& \psi_{i} \operatorname{sei} x_{i} /\{x\}, \\
& \varphi_{i+1} \operatorname{sei} \omega_{i} /(\{x\} \cup \square \cup C) .
\end{aligned}
$$

Die Bedingung (b) ist dann sicher erfüllt, weil

$$
\begin{aligned}
& Y_{i}=Y_{i-1} \cup\{x\}, \\
& X_{i+1}=Y_{i} \cup[, \\
& \left|V_{i} \psi_{i}\right|=1 .
\end{aligned}
$$

Dass auch die Bedingung (a) erfült ist, zeigen wir für beide Fälle 
gemeinsam. Erstens gilt wegen der Wahl von $x_{i}$ und $w_{i}$ :

$$
\begin{aligned}
\frac{1}{2} \cdot 4^{(p-i)} \cdot q & \leq \frac{1}{2} \cdot\left|X_{i} \backslash Y_{i-1}\right| \\
& =\frac{1}{2} \cdot(|A|+|C|+|E|) \\
& \leq|A|+|C| .
\end{aligned}
$$

Zweitens muss gelten $|A|<q$; andernfalls würde wegen unserer Bemerkung über Stemoperationen auf $s .20 \mathrm{mit} x_{i} / A$ als $\rho, w_{i} / A$ als $\sigma$ und $W=A$ der Fall (II) zutreffen. Daraus folgt nun aber:

$$
4^{p-(i+1)} \cdot q \leq \frac{1}{2} \cdot 4^{p-i} \cdot q-q \leq(|A|+|C|)-|A|=|C|=\left|x_{i+1} \backslash Y_{i}\right| .
$$

Ausserdem gilt mit * als $\underset{i+1}{*}$ :

$$
\begin{aligned}
& \varphi / x_{i+1} \geq\left(\psi_{0} * \cdots\left(\psi_{i-1} i_{i}^{*} \varphi_{i}\right) \ldots\right) / x_{i+1} \\
& \left(\psi_{0} \quad{ }_{1}^{*} \ldots\left(\psi_{i-1}{ }_{i}^{*}\left(x_{i} / x_{i+1} * \omega_{i} / x_{i+1}\right)\right) \ldots\right) \\
& \text { i.e. }\left(\psi_{0}{ }_{1}^{*} \cdots\left(\psi_{i-1}{ }_{i}^{*}\left(\psi_{i}{ }_{i+1}^{*} \varphi_{i+1}\right)\right) \ldots\right) \text {. }
\end{aligned}
$$

Es ist also auch die Bedingung (a) erfüllt.

Auf Grund der Definition unserer Formelfolge gilt nun insbesandere:

$$
\begin{aligned}
\varphi / x_{p} & \doteq\left(\psi_{0}{ }_{1}^{*} \ldots\left(\psi_{p-1} \stackrel{p}{p}_{p}\right) \ldots\right) \\
& \doteq\left(\psi_{1} \varphi^{*} \ldots\left(\psi_{p-1} \stackrel{p}{p}_{p}\right) \ldots\right), \text { und } \\
1 \leq q & \leq\left|x_{p}-Y_{p-1}\right| \leq\left|\operatorname{Var} \varphi_{p}\right| .
\end{aligned}
$$

Wir definieren nun noch zusätzlich eine Formel $\psi_{p}$ und eine Variablenmenge $V_{\uparrow}$ :

$$
\begin{aligned}
& V_{1}=\left\{\begin{array}{l}
Y_{p-1}, \text { falls } \operatorname{Var} \varphi_{p} \cap Y_{p-1} \neq \emptyset \\
Y_{p-1} \cup\{x\}, \text { falls } \operatorname{Var} \varphi_{p} \cap Y_{p-1}=\emptyset, \text { und } x \text { eine Variable aus }
\end{array}\right. \\
& \psi_{p} \text { sei } \varphi_{p} / V_{1} . \\
& \operatorname{Var} \varphi_{p} \text {. }
\end{aligned}
$$

Zusammenfassend erhalten wir also für $p=(k+1)^{(k \cdot r)}$ :

(1) $\varphi / v_{1} \geq\left(\psi_{1} 2_{2}^{*} \cdots\left(\psi_{p-1}{ }_{p}^{*} \psi_{p}\right) \ldots\right)$;

(2) für alle $i(1 \leq i \leq p): 1=\left|\operatorname{Var} \psi_{i}\right|$, oder $1<\left|\operatorname{Var} \psi_{i}\right|$ und $\operatorname{Var} \psi_{i} \subset \operatorname{Var}\left(\psi_{1} \cdot \ldots \cdot \psi_{i-1}\right)$;

(3) die bisher noch nicht verwendete voraussetzung $h\left(\varphi / V_{1}, V\right) \leq h(\varphi, V) \leq k$. 
2. Schritt Es seis $s=k \cdot r$, d.h. $p=(k+1)^{s}$. Wir beweisen mit Induktion nach $s(1 \leq s)$ :

Zu jeder natürlichen ZahI $p$, für welche $(1),(2),(3)$ und $(k+1)^{s} \leq p$ gilt, gibt es eine geeignete Variablenfolge $\left\langle v_{1}, \ldots, v_{s}\right\rangle$ aus $v_{1}^{5}$, Sternoperationen ${ }_{1}^{\prime}, . . . s_{s}^{*}$ und eine Formel $\omega^{\prime}$, sodass

$$
\begin{aligned}
& \left\{v_{1}\right\}=\operatorname{var} \psi_{1}, \\
& \varphi /\left\{v_{1}, \ldots, v_{s}\right\} ;\left(v_{1}{ }_{1}^{*}\left(\ldots\left(v_{s}^{*} s^{\prime} w^{\prime}\right) \ldots\right)\right) \text { und } \\
& h\left(w^{\prime}, v\right) \leq k-1 .
\end{aligned}
$$

Wir nennen $\left\langle v_{1}, \ldots, v_{s}\right\rangle$ genau dann eine geeignete Variablenfolge, wenn für alle $i_{1}, i_{2}, i_{3}$ gilt: Ist $1 \leq i_{1}<i_{2}<i_{3} \leq s$ und $v_{i_{1}}=v_{i_{3}}$, so ist auch $v_{i_{1}}=v_{i_{2}}$

Es sei $v_{1}$ die in $\psi_{1}$ vorkammende Variable.

Ist $s=1$, so gilt mit $\left(\psi_{2} 3^{*} \ldots\left(\psi_{p-1}{ }_{p}^{*} \psi_{p}\right) \ldots\right) /\left\{v_{1}\right\}$ als $\omega^{\prime}$ 'wegen unserer Bemerkung über sternoperationen auf $s .20$

$$
\varphi /\left\{v_{1}\right\} \geq\left(\psi_{1} 2_{2}^{*} \omega^{\prime}\right) \geq\left(v_{1}{ }_{1}^{*} \omega^{\prime}\right) ;
$$

ausserdem ist trivialerweise $h\left(\omega^{\prime}, v\right)=\ell\left(\omega^{\prime}, v_{q}\right) \leq k-1$.

Ist $1<s$, setzen wir $t=(k+1)^{(s-1)}+1$ und unterscheiden zwei fälle:

$v_{1}$ sei nicht enthalten in $v_{2}=\operatorname{Var}\left(\psi_{2} \cdot \ldots \cdot \psi_{t}\right)$ :

Wir wenden die Induktionsvoraussetzung an auf die folgende Formel, welche wir mit $\varphi^{\prime}$ bezeichnen:

$$
\left(\psi_{2}{ }_{3}^{*} \cdots\left(\psi_{t-1} t_{t}^{*} \psi_{t}^{\prime}\right) \ldots\right) \text {; }
$$

dabei sei $\psi_{t}^{\prime}$ die Formel $\left(\psi_{t}{ }_{t+1}^{*} \ldots\left(\psi_{p-1}^{*} \psi_{p}\right) \ldots\right) / V_{2}$.

Es gibt dann also ein geeignetes $\left\langle v_{2}, \ldots, v_{s}\right\rangle$ und ein $\omega^{\prime}$ mit:

$$
\begin{aligned}
& \varphi^{\prime} /\left\{v_{2}, \ldots, v_{s}\right\} \geq\left(v_{2} 2^{*} \ldots\left(v_{s}{ }_{s}, \omega^{\prime}\right) \ldots\right), \\
& h\left(\omega^{\prime}, v\right) \leq k-1 .
\end{aligned}
$$

Wegen unserer Bemerkung über Sternoperationen auf 5.20 gilt dann aber:

$$
\begin{aligned}
\varphi /\left\{v_{1}, \ldots, v_{s}\right\} & \vdots\left(\psi_{1} 2^{*} \varphi^{\prime} /\left\{v_{1}, \ldots, v_{s}\right\}\right) \\
& \vdots\left(v_{1} 1^{\prime} \varphi^{\prime} /\left\{v_{2}, \ldots, v_{s}\right\}\right) \\
& \left.\vdots\left(v_{1}\right)^{*}\left(v_{2} 2^{\prime} \ldots\left(v_{s}^{*}, \omega^{\prime}\right) \ldots\right)\right) ;
\end{aligned}
$$

dabei ist $h\left(\omega^{\prime}, v\right) \leq k-1$ und $\left\langle v_{1}, \ldots, v_{s}\right\rangle$ eine geeignete folge aus $v_{1}^{s}$.

$v_{1}$ sei enthalten in $\operatorname{Var}\left(\psi_{2} \cdot \ldots \cdot \psi_{t}\right)$ :

Wir setzen $h=\min \left\{j \mid v_{1}\right.$ in $\left.\operatorname{Var} \psi_{j}, 2 \leq j \leq t\right\}$ und

$v_{3}=\operatorname{Vvar}\left(\psi_{2} \cdots \cdots \psi_{h-1}\right)$. Es ist nun

$$
\phi v_{3} \geq\left(\psi_{1} / V_{3} 2^{*} \cdots\left(\psi_{p-1} / V_{3}{ }_{p}^{*} \psi_{p} / V_{3}\right) \ldots\right) .
$$


Von den Formeln $\psi_{i} / V_{3}(1 \leq i \leq p)$ enthalten dann $z$. B. noch die folgenden mindestens eine Variable:

$$
\psi_{1} / V_{3}, \psi_{h} / V_{3}, \psi_{h_{2}} / V_{3}, \ldots, \psi_{h_{p}}, N \quad\left(h<h_{2}<h_{p}, \leq p\right) .
$$

Wir definieren nun formeln $\omega_{0}, \omega_{1}, \ldots, \omega_{p}$, wie folgt:

$\omega_{0} \operatorname{sei} \psi_{1} / N_{3}$.

$\omega_{1}$ sei $\psi_{h} / v_{3}$, d.h. dass $\operatorname{Var} \omega_{1}=\operatorname{Var} \omega_{0}=\left\{v_{1}\right\}$,

$\omega_{j}$ sei $\psi_{h} / V_{3}$, für $2 \leq j \leq p^{\prime}-1$,

$\omega_{p^{\prime}} \operatorname{sei}\left(\psi_{h_{p^{\prime}-1}+1} / V_{3} h_{p^{\prime}-1}^{*}+2 \cdots\left(\psi_{p-1} / V_{3}{ }_{p}^{*} \psi_{p} / V_{3}\right) \ldots\right)$.

Wegen unserer Bemerkung über Sternoperationen auf $S .20$ ist

$$
\varphi / v_{3} \geq\left(\omega_{0}{ }_{1}^{* *}\left(\omega_{1} 2^{*} \cdots\left(\omega_{p},-1, p_{p}^{*} \omega_{p}, \ldots\right)\right)\right.
$$

und ausserdem gelten für $\left(\omega_{1} 2^{*} \ldots\left(\omega_{p},-1 p_{p}^{*}, \omega_{p}, \ldots\right)\right.$ die Bedingungen (1), (2), (3). Um auf diese Formel (wir nennen sie $\varphi^{\prime}$ ) die Induktionsvoraussetzung anwenden zu können, müssen wir noch zeigen, dass $(k+1)^{s-1} \leq p^{\prime}$.

Die Variablenmenge $\operatorname{Var}\left(\psi_{2} \cdot \ldots \cdot \psi_{h-1}\right)$ enthält wegen (2) höchstens $h-2$ Variable, von denen wegen (3) jede höchstens $k-1$ mal in

$\left(\psi_{h} h^{*} \cdots\left(\psi_{p-1}{ }_{p}^{*} \psi_{p}\right) \ldots\right)$ vorkommt. Deshalb enthalten mindestens noch $p-((h-2)+(h-2)(k-1))$ der Formeln $\psi_{j} / V_{3}(1 \leq j \leq p)$ eine Variable. Das heisst:

$$
p-((h-2)+(h-2)(k-1))=p-(h-2) \cdot k \leq p^{\prime}+1 .
$$

Damit gilt nun aber:

$$
\begin{aligned}
(k+1)^{s-1}+1 & \leq(k+1)^{s-1}+k \\
& =(k+1)^{s}-\left((k+1)^{s-1}-1\right) \cdot k \\
& \leq p-(t-2) \cdot k \\
& \leq p-(h-2) \cdot k \\
& \leq p^{\prime}+1 .
\end{aligned}
$$

Wird nun die Induktionsvoraussetzung auf $\varphi^{\prime}$ angewandt, bedeutet das: Es gibt ein geeignetes $\left\langle v_{2}, \ldots, v_{s}\right\rangle$, Sternoperationen $2_{2}^{*} \ldots{ }_{s}^{*}$ und eine Formel $\omega^{\prime}$, sodass

$$
\begin{aligned}
& \varphi^{\prime} /\left\{v_{2}, \ldots, v_{s}\right\} \geq\left(v_{2}{ }_{2}^{*} \ldots\left(v_{s} *_{s}^{\prime} \omega^{\prime}\right) \ldots\right) . \\
& h\left(\omega^{\prime}, v\right) \leq k-1 \text {, und } \\
& \left\{v_{2}\right\}=\operatorname{var} \omega_{1}, \text { d.h. } v_{1}=v_{2} \cdot
\end{aligned}
$$

Deshalb, und wegen unserer Bemerkung über Sternoperationen auf $S .20$ ist dann aber

$$
\begin{aligned}
\varphi /\left\{v_{1}, \ldots, v_{s}\right\} & \vdots\left(\omega_{0}{ }_{1}^{* \prime} \varphi^{\prime} /\left\{v_{2}, \ldots, v_{s}\right\}\right) \\
& \vdots\left(v_{1}{ }_{1}^{*}\left(v_{2} 2^{*} \ldots\left(v_{s}^{*} s^{*} w^{\prime}\right) \ldots\right),\right.
\end{aligned}
$$


wo $h\left(\omega^{\prime}, V\right) \leq k-1$.

$\left\langle v_{1}, v_{2}, \ldots, v_{s}\right\rangle$ ist eine geeignete Folge, weil $\left\langle v_{2}, \ldots, v_{5}\right\rangle$ eine geeignete Folge ist, und weil $v_{1}=v_{2}$.

3. Schritt weil $h(\varphi, v) \leq k$, und $s=k \cdot r$, enthält $\left\{v_{1}, \ldots, v_{s}\right\}$ mindestens $r$ verschiedene Variable $w_{1}, \ldots, w_{r}$. Auf Grund der folgenden Eigenschaften der Sternoperationen:

$$
\begin{aligned}
& \left(v{ }_{1}^{*}\left(v{ }_{2}^{*} \varphi\right)\right) \text { 茄 }\left(v{ }_{3}^{*} \varphi\right) \\
& \left(0{ }_{1}^{*}\left(\varphi{ }_{2}^{*} \psi\right)\right) \geq\left(\varphi{ }_{4}^{*} \psi\right),
\end{aligned}
$$

gibt es eine formel $w$ und Sternoperationen ${ }_{1}^{*} \ldots{ }^{*}$, sodass $\varphi /\left\{w_{1}, \ldots, w_{r}\right\}$ ä口 $\left(w_{1}{ }_{1}^{*}\left(w_{2} 2_{2}^{\bar{*}} \ldots\left(w_{r}{ }_{r}^{\bar{*}} w\right) \ldots\right)\right.$,

und $h(\omega, V) \leq k-1$.

Damit wäre des Lemma 1 bewiesen.

\section{Normale Farme in}

Bezugnehmend auf die Interpretation in einem endlichen kommutativen Ring $k$ mit Einselement und Universum $K$, wollen wir gewisse Formeln aus $\Phi(V, K)$ auszeichnen und "normal" nennen. Wir definieren

- die Menge $H(x, k)$ der homogenen Formeln in der Variablen $x$ :

$$
\begin{aligned}
& \varphi \text { gehöre genau dann zu } H(x, k), \\
& \text { wenn } \varphi \text { aus } \Phi(x, k) \text { und }\left.\varphi\right|_{D} ^{x} \text { äq } 0 .
\end{aligned}
$$

- die Menge $S(w, \hat{k})$ der Summenformeln, und die Menge $P(w, k)$ der Produktformeln über einer Teilmenge $W$ von $V$ :

$$
\begin{aligned}
& \text { } \varphi \text { gehöre genau dann zu } S(w, k) \text { bzw. } P(w, k) \text {, } \\
& \text { wenn es ein homogenes } \psi \text { gibt, sodass } \varphi \\
& \text { die Formel }\left.\sum_{w \in W} \psi\right|_{w} ^{x} \text { bzw. } \prod_{w \in W}\left(1+\left.\psi\right|_{w} ^{x}\right) \text { ist. }
\end{aligned}
$$

N.B. Sind $z \cdot B$. $w_{1}, w_{2}, w_{3}, w_{4}$ die verschiedenen Elemente von $w$, so bezeichnen wir mit $\sum_{w \in W} w$ die Formel $\left(w_{1}+\left(w_{2}+\left(w_{3}+w_{4}\right)\right)\right)$.

- die Menge $G(W, k)$ der Grundformeln über $W$ :

$$
G(w, \bar{k})=\Phi(S(w, \hat{k}) \cup P(w, \bar{k}), k) \text {. }
$$

- die Menge $N(V, k)$ der normelen Formeln aus $\Phi(V, K)$ :

$$
N(V, \bar{k})=\bigcup_{W=V} G(W, \bar{k}) \text {. }
$$

Bemerkung Ist $k$ der Körper $k_{0}$ mit dem Universum $\{0,1\}$, so ist jede in $\times$ homogene formel äquivalent

$$
x \text { oder } 0 \text {, }
$$

jede Summenformel über W gaquivalent 


$$
0 \text { oder } \sum_{w \in W} w,
$$

jede Produktformel über $W$ äquivalent

$$
1 \text { oder } \prod_{w \in W}(1+w) \text {, }
$$

und jede Grundformel über $W$ äquivalent einer Formel

$$
\left(+\left(\left(b \cdot \sum_{w \in W} w\right)+\left(c \cdot \prod_{W \in W}(1+w)\right)\right)\right),
$$

wo $a, b, c$ aus $\{0,1\}$ sind.

Bemerkung Für Formeln $\varphi$, $\psi$ und Teilmengen $W$, $W$ ' von $V$ gilt:

(i) Alle Konstanten sind Grundformeln.

(ii) Sind $\varphi$ und $\psi$ Grundformeln über $W$, so auch $\left(\varphi^{*} \psi\right)$.

(iii) Ist $\varphi$ eine Grundformel über $W$, und $W^{\prime} \subset W$, so ist $\varphi / W^{\prime}$ eine Grundformel über W'. D.h. die Menge der normalen Formeln ist abgeschlossen gegenüber dem Nullsetzen von Variablen.

(iv) Ist $\varphi$ eine Grundformel über $W$, so ist $\hat{\varphi}(k)$ eine symmetrische Abbildung von $K^{W}$ in $K$.

\section{Lemma 2}

Es sei $k$ der Körper $k_{0}$ mit dem Universum $K=\{0,1\}$. Für jede Variablenmenge $V$, jede Formel $\varphi$ aus $\Phi(V, K)$ und jede natürliche Zahl $t$ gilt dann:

Gibt es alles verschiedene Variablen $w_{1}, \ldots, w_{5 t}$ und eine formel $w$, sodass $\varphi /\left\{w_{1}, \ldots, w_{5 t}\right\}$ äq $\left(w_{1} \overline{1}_{1} \ldots\left(w_{5 t} \overline{5}^{*}(w) \ldots\right)\right.$, so gibt es eine Teilmenge $Z$ van $\left\{w_{1}, \ldots, w_{5 t}\right\}$, eine Grundformel $\psi$ über $z$, eine Sternoperation * und eine formel $\tau$, sodass $|z|=t, \varphi / Z$ äq $(\psi * \tau)$, und $h(\tau, V) \leq h(\omega, V)$.

Beweis Bezüglich $\tilde{k}_{0}$ gilt für jede Sternoperation:

$$
(x * \varphi) \text { äq }((a+(b \cdot x))+((c+(d \cdot x)) \cdot \varphi)) .
$$

D.h. es gibt nur die folgenden Typen von Sternoperationen:

$$
\begin{array}{ll}
\text { "Null-Sterne": } & \left(x^{*} \varphi\right) \text { äq }((a+(b \cdot x))+((d \cdot x) \cdot \varphi)), \\
\text { "A-Sterne": } & (x * \varphi) \text { äq }((a+(b \cdot x))+\varphi), \\
\text { "B-Sterne": } & \left(x^{*} \varphi\right) \text { äq }(a+((1+x) \cdot \varphi), \\
\text { "C-Sterne": } & \left(x^{*} \varphi\right) \text { äq }((a+1)+((1+x) \cdot(1+\varphi))) .
\end{array}
$$

Dabei sind $a, b, c$ und $d$ Elemente von $\{0,1\}$.

Zu jedem C-Stern ${ }_{1}^{*}$ gibt es einen B-Stern ${ }_{1}^{*}$, sodass

$$
\left(\varphi{ }_{1}^{*}\left(\psi \psi_{2}^{*} \chi\right)\right) \text { äq }\left(\varphi_{1}^{*}\left(\psi \psi_{2}^{*}, x\right)\right) \text {. }
$$

Es gibt darum Sternoperationen ${ }_{1}^{*}, \ldots$. $_{5}^{*}$ unter denen keine C-Sterne sind, und eine formel $\tau_{1}$, sodass 


$$
\varphi /\left\{w_{1}, \ldots w_{5 t}\right\} \text { 茄 }\left(w_{1}{ }_{1}^{*} \ldots\left(w_{5 t}{ }_{5 t}^{*} \tau_{1}\right) \ldots\right) \text {, }
$$

dabei ist $\tau_{1}$ entweder $w$ oder $(1+\omega), d . h$. $h\left(\tau_{1}, V\right)=h(w, V)$.

Fall 1 Bei ${ }_{1}^{*}, \ldots *_{4 t}^{*}$ sei ein Null-Stern dabei.

Für $Z=\left\{w_{4 t+1}, \ldots, w_{5 t}\right\}$ gilt dann: $|z|=t$, und $\varphi / z$ ist äquivalent einer Konstanten, d.h. zum Beispiel àquivalent $\left(c_{1} * c_{2}\right)$.

Fall 2 Bei ${ }_{1}^{*}, \ldots * * 4_{t}^{*}$ sei kein Null-Stern dabei. Die Anzahl der A-Sterne darunter sei $n_{A}$, die Anzahl der B-Sterne darunter sei $n_{B}$; es ist also $n_{A}+n_{B}=4 t$. Für $Z_{1}=\left\{w_{1}, \ldots, w_{4 t}\right\}$ gibt es ein $\tau_{2}$, sodass

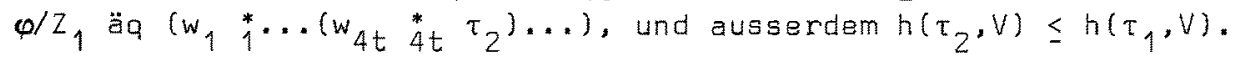

Fall 2.1 Es sei $2 t \leq n_{A} ; z_{2}=\left\{z_{1}, \ldots, z_{2 t}\right\}$ sei eine Teilmenge von $\left\{w_{i} \mid 1 \leq i \leq 4 t,{ }_{i}^{*}\right.$ ist ein A-Stern $\}$ mit $\left|z_{2}\right|=2 t$. Es ist dann

$$
\varphi / z_{2} \text { äq }\left(e+\left(\sum_{i=1}^{2 t}\left(b_{i} \cdot z_{i}\right)+\tau_{2}\right)\right) \text {. }
$$

Es sei nun $\tau$ die Formel $\tau_{2}$, und $Z$ eine $t$-elementige teilmenge einer der beiden folgenden Mengen

$$
\begin{aligned}
& \left\{z_{i} \mid 1 \leq i \leq 2 t, b_{i}=0\right\} \\
& \left\{z_{i} \mid 1 \leq i \leq 2 t, b_{i}=1\right\} .
\end{aligned}
$$

Dann ist $\varphi / Z$ äquivalent $(e+\tau)$ oder $\left(\left(e+\sum_{z \in Z} z\right)+\tau\right)$, und $h(\tau, V)=h\left(\tau_{2}, V\right) \leq h\left(\tau_{1}, V\right)=h(\omega, V) . \quad z \in Z$

Fall 2.2 Es sei $2 t \leq n_{B} ; z_{2}=\left\{z_{1}, \ldots, z_{2 t}\right\}$ sei eine Teilmenge von $\left\{w_{i} \mid 1 \leq i \leq 4 t,{ }_{i}^{*}\right.$ ist ein B-Stern $\}$ mit $\left|z_{2}\right|=2 t$.

Es j.st dann z.B.

$$
\varphi / z_{2} \text { äq }\left(e_{1}+\left(\left(1+z_{1}\right) \cdot\left(e_{2}+\left(\left(1+z_{2}\right) \cdot \ldots \cdot\left(e_{2 t}+\left(\left(1+z_{2 t}\right) \cdot\left(e+\tau_{2}\right)\right)\right) \ldots\right)\right)\right)\right.
$$

wir definieren nun Formeln $\pi_{1}, \pi_{2}, \ldots, \pi_{2 t+1}$;

Falls für alle $i(2 \leq i \leq 2 t) e_{i}=0$, seien

$$
\begin{gathered}
\pi_{1} \text { die Formel } \prod_{i=1}^{2 t}\left(1+z_{i}\right) \text {, und } \\
\pi_{2}, \pi_{3}, \ldots, \pi_{2 t+1} \text { alle die Formel } 1 .
\end{gathered}
$$

Falls $e_{i}=1$ genau denn, wenn $i=i_{1}, i_{2}, \ldots, i_{h}\left(2 \leq i_{1}<\ldots<i_{h} \leq 2 t\right)$, seien 


$$
\begin{gathered}
\pi_{1} \text { die Formel } \prod_{i=1}^{i_{1}^{-1}}\left(1+z_{i}\right), \\
\pi_{2} \text { die Formel } \prod_{i=i}^{i_{2}-1}\left(1+z_{i}\right), \\
\pi_{h+1} \text { die Formel } \prod_{i=i_{h}}^{2 t}\left(1+z_{i}\right), \\
\pi_{h+2}, \ldots, \pi_{2 t+1} \text { alle die Forme } 1 \text {. }
\end{gathered}
$$

Es ist dann für irgendein $h(0 \leq h \leq 2 t-1)$ :

$$
\varphi / z_{2} \text { ãq }\left(\varepsilon_{1}+\left(\pi_{1} \cdot\left(1+\left(\pi_{2} \cdot \ldots \cdot\left(1+\left(\pi_{h+1} \cdot\left(e+\tau_{2}\right)\right)\right) \ldots\right)\right)\right) .\right.
$$

Ist $\tau$ die Formel $\tau_{2}$ oder die Formel $\left(1+\tau_{2}\right)$, und $z$ eine t-elementige Teilmenge einer der beiden folgenden Mengen

so ist

$$
\begin{aligned}
& \operatorname{Var}\left(\pi_{1} \cdot \pi_{3} \cdots \pi_{2 t+1}\right) \\
& \operatorname{Var}\left(\pi_{2} \cdot \pi_{4} \cdots \pi_{2 t}\right),
\end{aligned}
$$

$$
\begin{aligned}
& \varphi / z \text { äq }\left(e^{\prime}+\left(\prod_{z \in Z}(1+z) \cdot \tau\right)\right) \\
& \text { und } \\
& h(\tau, V)=h\left(\tau_{2}, V\right) \leq h\left(\tau_{1}, V\right)=h(\omega, V) .
\end{aligned}
$$

Damit wäre auch das Lemma 2 bewiesen.

Um die beiden nachfolgenden Sätze einfacher formulieren zu können, definieren wir zwei zahlentheoretische Funktionen:

Für alle natürlichen Zahlen $m$ sei

$$
\begin{aligned}
& \theta(0, m)=m, \\
& \text { und für } k=1,2, \ldots: \\
& \begin{aligned}
\theta(k, m) & =\Gamma(k, m, \theta(k-1, \theta(k-1, m)), 5 \cdot \theta(k-1, m)) \\
& =4^{(k+1)^{5 k \cdot \theta(k-1), m)}-1} \cdot \theta(k-1, \theta(k-1, m))+m .
\end{aligned}
\end{aligned}
$$

Für alle natürlichen Zahlen $c$ und $m$ sei

$$
\Delta(\mathrm{c}, \mathrm{m})=2 \cdot \theta(2 \mathrm{c}, \mathrm{m}) \text {. }
$$

\section{Satz 1}

Es sei $k$ der Kärper $k_{0}$ mit dem Universum $K=\{0,1\}$. Für jede nicht-negative ganze Zahl $k$, jede natürliche Zahl $\mathrm{m}$, jede Variablenmenge $V$ und jede Formel $\varphi$ aus $\Phi(V, K)$ gilt:

Ist $\theta(k, m) \leq|V|$ und $h(\varphi, V) \leq k$, so gibt es eine Teilmenge $U$ von $V$ und 
eine Grundformel $\psi$ über $U$, sodass $|U|=m$ und $\varphi / U$ äq $\psi$.

Beweis mit Induktion nach $k$. Ist $k=0$, so gilt $m \leq|V|$ und $h(\varphi, V)=0$. D.h. es gibt eine m-elementige Teilmenge $U$ von $V$, und $\varphi / U$ ist äquivalent einer Konstanten:

Ist $0<k$, so gilt nach Lemma 1:

$$
\begin{aligned}
& \text { (I), oder } \\
& \text { (II) mit } q=\theta(k-1, \theta(k-1, m)) \text {, oder } \\
& \text { (III) mit } r=5 \cdot \theta(k-1, m) \text {. }
\end{aligned}
$$

Im Fall (I) sind wir fertig mit $U=W$.

Im Fall (II) gilt für $U_{1}=\operatorname{Var}_{\rho} \cap$ Varo:

$$
\begin{aligned}
& \varphi / U_{1} \geq\left(\rho / U_{1} * \sigma / U_{1}\right), \\
& \theta(k-1, \theta(k-1, m)) \leq\left|U_{1}\right|, \\
& h\left(\rho / U_{1}, V\right) \leq k-1 \text { und } h\left(\sigma / U_{1}, V\right) \leq k-1 .
\end{aligned}
$$

Nach Induktionsvoraussetzung gibt es eine Teilmenge $U_{2}$ von $U_{1}$ und eine Grundforme 1 über $U_{2}$, sodass

$$
\begin{aligned}
& \rho / U_{2} \text { äq } x . \\
& \theta(k-1, m)=\left|u_{2}\right|, \text { und } \\
& h\left(\sigma / U_{2}, v\right) \leq k-1 .
\end{aligned}
$$

Nach Induktionsvoraussetzung gibt es eine Teilmenge $U$ von $U_{2}$ und eine Grundformel w über $U$, sodass

$$
\sigma / U \text { 茄 } \omega \text {, und } m=|U| \text {. }
$$

Es ist darum

$$
\begin{aligned}
& \varphi / U \geq(\rho / U * \sigma / U) \\
& \text { ăq }(\chi / U * \omega) ;
\end{aligned}
$$

$(\chi / U * \omega)$ ist aber sicher eine Grundformel über $U$.

Im Fall (III) wenden wir das Lemma 2 an mit $t=\Theta(k-1, m)$. Danach gibt es eine Teilmenge $Z$ von $V$, eine Grundformel $\psi$ über $Z$, eine Sternoperation * und eine Formel $\tau$, sodass

$$
\begin{aligned}
& |z|=t=\theta(k-1, m), \\
& \varphi / z \text { äq }(\psi * \tau), \text { und } \\
& h(\tau, v) \leq k-1 .
\end{aligned}
$$

Nach Induktionsvoraussetzung gibt es eine Teilmenge $U$ von $Z$ und eine Grundformel $\omega$ über $U$, sodass

$$
\tau / U \text { äq } \omega \text {, und } m=|U| \text {. }
$$

Es ist darum

$$
\varphi / \cup \text { äq }(\psi / U * \omega) \text {; }
$$

$(\psi / U * \omega)$ ist aber sicher eine Grundformel über $U$. 


\section{Satz 2}

Es sei $k$ der Körper $k$ mit dem Universum $K=\{0,1\}$. Für alle natürlichen Zahlen $c$ und $m$, jede Variablenmenge $V$ und jede Formel $\varphi$ sus $\Phi(V, K)$ gilt:

Ist $\Delta(\mathrm{c}, \mathrm{m}) \leq|\mathrm{V}|$ und $\ell(\varphi, V) \leq c \cdot|V|$, so gibt es eine Teilmenge $U$ von $V$ und eine Grundformel $\psi$ über $U$, sodass $|U|=m$, und $\varphi / U$ äq $\psi$.

Beweis Nach Voraussetzung ist

$$
\begin{aligned}
& \Delta(c, m)=2 \cdot \theta(2 c, m) \leq|V| . \\
& \ell(\varphi, v) \leq c \cdot|V| .
\end{aligned}
$$

Wir zerlegen die Variablenmenge $V$ :

Es gilt dann folgendes:

$$
\begin{aligned}
& x_{1}=\{x \in V \mid 20<\ell(\varphi, x)\} \\
& x_{2}=\{x \in V \mid \&(\varphi, x) \leq 2 c\} .
\end{aligned}
$$

$$
\begin{aligned}
& \left|x_{1}\right|+\left|x_{2}\right|=|v| \\
& 2 c \cdot\left|x_{1}\right|<\ell\left(\varphi, x_{1}\right) \leq \ell(\varphi, v) \leq c \cdot|v| . \\
& \theta(2 c, m) \leq \frac{|v|}{2}=|v|-\frac{|v|}{2}<|v|-\left|x_{1}\right|=\left|x_{2}\right|, \\
& h\left(\varphi / x_{2}, x_{2}\right) \leq 20 .
\end{aligned}
$$

Nach Satz 1 gibt es dann eine Teilmenge $U$ von $x_{2}$ und eine Grundformel $\psi$ über $U$, sodass $|U|=m$ und $\varphi / U$ äq $\psi$.

\section{Verallgemeinerung}

Im Folgenden soll gezeigt werden, dass im Falle $k \neq k_{0}$ keine dem Lemma 2, dem Satz 1 und dem Satz 2 entsprechenden Sätze gelten. Wir zeigen das für den Satz 1 , indem wir folgendes beweisen:

Ist $k \neq k_{0}$, so gibt es zu jeder natürlichen Zahl $t$ eine Variablenmenge $V$ und eine Formel $\varphi$ aus $\Phi(V, K)$ mit:

$$
\begin{aligned}
& |V|=t, \\
& h(\varphi, V)=3 \text {, und } \\
& \text { ist } W \text { eine Teilmenge von } V \text { mit } 2 \leq|W|, \\
& \text { so ist } \varphi / W \text { nicht äquivalent einer } \\
& \text { Grundformel uber } W .
\end{aligned}
$$

Es genügt den Beweis durchzuführen für Teilmengen $U$ von $V$ mit $|U|=2$; denn ist $U C W$ und $\varphi / U$ nicht äquivalent einer Grundformel über $U$, so ist $\varphi / W$ nicht äquivalent einer Grundformel über W. Es sei also 


$$
\begin{aligned}
& v=\left\{v_{1}, \ldots, v_{t}\right\}, \\
& \varphi \text { die Forme } 1\left(v_{t}+\left(1+\left(v_{t} \cdot v_{t}\right)\right) \cdot \ldots\left(v_{2}+\left(1+\left(v_{2} \cdot v_{2}\right)\right) \cdot v_{1}\right) \ldots\right) \text {. und } \\
& U=\left\{u_{1}, u_{2}\right\} \subset v .
\end{aligned}
$$

Es ist dann z.B. $\varphi /$ U die Formel $\left(u_{2}+\left(\left(1+\left(u_{2} \cdot u_{2}\right)\right) \cdot u_{1}\right)\right)$. Ist aber $K \neq \tilde{k}_{0}$, so gibt es $c_{1}$ und $c_{2}$ aus $K$, sodass

$$
\begin{aligned}
c_{2}^{2} \cdot c_{1} & \neq c_{2} \cdot c_{1}^{2} \\
c_{2}+\left(1+c_{2}^{2}\right) \cdot c_{1} & \neq c_{1}+\left(1+c_{1}^{2}\right) \cdot c_{2}
\end{aligned}
$$

$\varphi / U$ stellt also keine symmetrische Abbildung von $K^{U}$ in $K$ dar und ist deshalb nicht äquivalent einer Grundformel über $U$.

\section{Anwendung}

Im Fall $k=k_{0}$ sollen hier einige Gegenbeispiele zur folgenden Behauptung konstruiert werden:

Es gibt eine natürliche Zahl $c$, sodass es für alle Variablenmengen $V$ und alle Abbildungen $f$ von $\{0,1\}^{V}$ in $\{0,1\}$ eine Formel $\varphi$ aus $\Phi(V)$ gibt mit :

$\varphi$ stelit $f$ dar, und

$$
\ell(\varphi, V) \leq c \cdot|V| \text {. }
$$

(Wir schreiben hier wie im Aufsatz über den Satz von Netschiporuk für $\Phi(V,\{0,1\})$ kürzer $\Phi(V))$.

Zur Konstruktion dieser Gegenbeispiele definieren wir zuerst spezielle Folgen von symmetrischen Abbildungen:

Es sei $n$ eine natürliche $Z$ ah 1 , und $V_{n}$ die Menge der Variablen $x_{j}$ mit $1 \leq j \leq n$. Für jede Teilmenge $Q$ von $\{0,1,2, \ldots\}$ definieren wir eine Folge von Abbildungen $\left\langle f_{Q}^{1}, f_{Q}^{2}, \ldots>\right.$ mit:

$f_{Q}^{n}$ ist eine Abbildung von $\{0,1\}^{V_{n}}$ in $\{0,1\}$. Ist b eine Abbildung von $V_{n}$ in $\{0,1\}$, und $r_{b}$ die Anzahl der natürlichen Zahlen $j$ mit $1 \leq j \leq n$ und $b\left(x_{j}\right)=1$, so ist genau dann $f_{Q}^{n}(b)=1$, wenn $\Gamma_{b}$ in $Q$.

Bemerkung Es gibt Folgen von symmetrischen Abbildungen

$$
f_{n}:\{0,1\}^{V_{n}} \Longrightarrow\{0,1\} \quad(n=1,2, \ldots)
$$

welche nicht von dem eben definierten Typ sind.

1. Beispiel $f_{n}(b)=1$ genau dann, wenn $r_{b}=n$. Diese Abbildungen sind darstellbar durch $\left(x_{1} \cdot\left(x_{2} \cdot \ldots\left(x_{n-1} \cdot x_{n}\right) \ldots\right)\right)$.

2. Beispiel Es sei $f_{n}(b)=1$ genau dann, wenn $r_{b} \neq n$ (modulo 2). Diese Abbildungen sind darstellbar durch $\left(\left(1+x_{1}\right)+\left(\left(1+x_{2}\right)+\ldots+\left(1+x_{n}\right) \ldots\right)\right)$. 
Wir betrachten nun spezielle Teilmengen von $\{0,1,2, \ldots\}$ :
$Q_{1}=\emptyset$
$Q_{5}=\{0,1,2, \ldots\}$
$Q_{2}=\{0\}$
$Q_{6}=\{1,2,3, \ldots\}$
$Q_{3}=\{1,3,5, \ldots\}$
$Q_{7}=\{0,2,4, \ldots\}$
$Q_{4}=\{0,1,3,5, \ldots\}$
$Q_{8}=\{2,4,6, \ldots\}$

Für die n-ten Abbildungen der zugehörigen Folgen gibt es in $\Phi\left(V_{n}\right)$ die folgenden Darstellungen (in abgekürzter Schreibweise):

$$
\begin{array}{ll}
f_{Q_{1}}^{n}: 0 & f_{Q_{5}}^{n}: 1 \\
f_{Q_{2}}^{n}: \prod_{i=1}^{n}\left(1+x_{i}\right) & f_{Q_{6}}^{n}: 1+\prod_{i=1}^{n}\left(1+x_{i}\right) \\
f_{Q_{3}}^{n}: \sum_{i=1}^{n} x_{i} & f_{Q_{7}}^{n}: 1+\sum_{i=1}^{n} x_{i} \\
f_{Q_{4}}^{n}: \prod_{i=1}^{n}\left(1+x_{i}\right)+\sum_{i=1}^{n} x_{i} & f_{Q_{8}}^{n}: 1+\prod_{i=1}^{n}\left(1+x_{i}\right)+\sum_{i=1}^{n} x_{i}
\end{array}
$$

Diese Folgen sind also keine Gegenbeispiele zur eingangs formulierten Behauptung.

Es bedeute nun $O(Q)$, dass $Q$ eine Teilmenge von $\{0,1,2, \ldots\}$ und verschieden von $Q_{1}, Q_{2}, \ldots, Q_{\theta}$ ist. Man sieht sofort, dass $Q(Q)$ gleichbedeutend ist mit:

$Q$ ist eine Teilmenge von $\{0,1,2, \ldots\}$, und es gibt zwei Zahlen $p$ und $q$ aus $\{1,2,3, \ldots\}$ mit: $p=q(\operatorname{modulo} 2)$, und $p$ nicht aus $Q, q$ aus $Q$.

Satz Für jedes $Q$ mit $\boldsymbol{a}(Q)$ kann zu jeder natürlichen Zahl c eine natürliche Zahl $n$ angegeben werden, sodass für jede Formel $\varphi$ aus $\Phi\left(V_{n}\right)$, welche $f_{Q}^{n}$ darstellt, gilt:

$$
c \cdot\left|v_{n}\right|=c \cdot n<\ell\left(\varphi, v_{n}\right)
$$

Mit der zahlentheoretischen Funktion $\Delta$ aus Satz 2 gibt man $n$ wie folgt an:

$$
n=\Delta(c, \max (p, q))
$$

Beweis Es sei $p=q(\bmod 2), p \notin Q, q \in Q$ und $n$ wie eben angegeben definiert. Ist nun $\varphi$ eine Formel aus $\Phi\left(V_{n}\right)$ mit $\ell\left(\varphi, V_{n}\right) \leq c \cdot n=c \cdot\left|v_{n}\right|$, so falgt nach Satz 2 :

Es gibt eine Teilmenge $U$ von $V_{n}$ und eine Grundformel $\psi$ über $U$, sodass

$$
|u|=\max (p, q) \text {, und } \varphi / U \text { äq } \psi \text {. }
$$


In unserm Fall bedeutet das, dass $\psi$ äquivalent

$$
A+B \cdot \prod_{w \in U}(1+w)+c \cdot \sum_{w \in U} w,
$$

wo $A, B$ und $C$ Konstante aus $\{0,1\}$ sind.

Es sei nun $Z$ eine Teilmenge von $U \operatorname{mit}|Z|=\min (p, q)$. $|U \backslash Z|$ ist dann gerade, und $1 \leq|z|$. Wir definieren zwei Abbildungen b und $b^{\prime}$ von $v_{n}$ in $\{0,1\}:$

$$
\begin{aligned}
& b(w)=1, \text { für w aus } z, \\
& b(w)=0, \text { für } w \text { aus } v_{n} \cdot z ; \\
& b^{\prime}(w)=1, \text { für } w \text { aus } u^{\prime} \\
& b^{\prime}(w)=0, \text { für } w \text { aus } v_{n} \backslash .
\end{aligned}
$$

Es ist dann $\hat{\varphi}(b)=\phi(b)=\phi\left(b^{\prime}\right)=\hat{\varphi}\left(b^{\prime}\right)$.

Anderseits ist aber $r_{b}=\min (p, q)$ und $r_{b}$, $=\max (p, q)$.

Darum gilt:

$$
\begin{aligned}
& r_{b} \in Q \text { und } r_{b}, \notin Q \text {, oder } \\
& r_{b} \notin Q \text { und } r_{b}, \in Q \text {. }
\end{aligned}
$$

Das heisst, dass $f_{Q}^{n}(b) \neq f_{Q}^{n}\left(b^{\prime}\right)$; womit gezeigt ist, dess $f_{Q}^{n}$ nicht durch $\varphi$ dargestelit wird.

Beispiel Es sei $Q=\{0,1,2, \ldots\} \backslash\{0,1\}$. Wegen $1 \notin Q, \exists \in Q$ und $1=3$ (mod 2) gilt $a(Q)$. $f_{Q}^{n}$ wird (in abgekürzter Schreibweise) dargestellt durch die Formel

$$
1+\prod\left(1+x_{i} \cdot x_{j}\right), \quad(1 \leq i \quad j \leq n) .
$$

Unser Satz besagt nun:

Für alle natürlichen Zahlen $c$ und $n$ mit $n=\Delta(c, 3)$ hat jede Formel aus $\Phi\left(V_{n}\right)$, welche $f_{Q}^{n}$ darstellt, eine Länge grösser als $c \cdot n$.

\section{Literetur}

Fischer, M.J. and Meyer, A.R. and Paterson, M.S., Lower bounds on the size of Boolean formulas: Preliminary report. Paper to be presented at $7^{\text {th }}$ ACM Symp. On Theory of Computing, May 1975.

Harper, L.H. and Savage, J.E., On the complexity of the marriage problem, Adv. in Math. 9, 3(1972) 299-312.

Hodes, L. and Speoker, E., Lengths of formulas and elimination of quantifiers I, Contr. to Math. Log. (1968) 175-188. 
Něciporuk, E.I., A Boolean function, Dokl. Akad. Nauk SSSR 169 (1966) 765-766, Engl. Trans1.: Soviet Math. Dokl. 7 (1966) 999-1000. Subbotovskaja, B.A., Comparison of bases in the realization by formulas of functions of the algebra of logic. Dokl. Akad. Nauk SSSR 149 (1963) 784-787, Engl. trans 1.: Soviet Math. Dok1. 4 (1963) 478-481. 